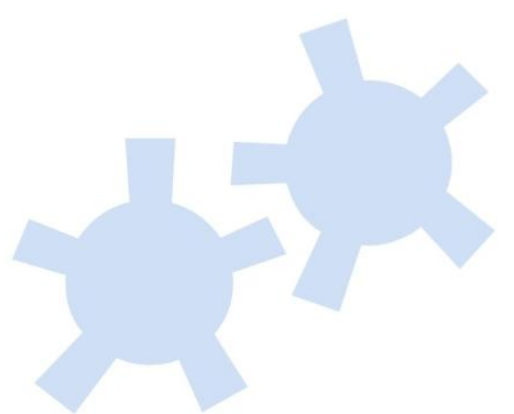

Sar: Automatic Generation of Statistical Reports Using Stata and Microsoft Word for Windows

Giovanni Luca Lo Magno

lomagno.gl@virgilio.it

Department of Economics, Business and Finance

University of Palermo 


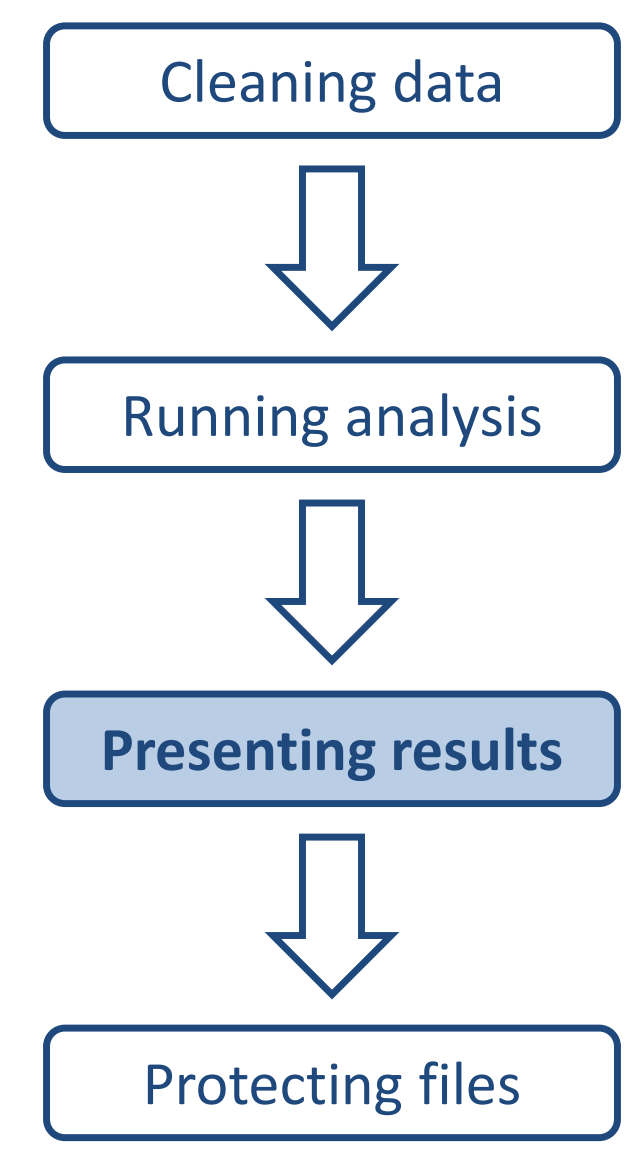


Automatic
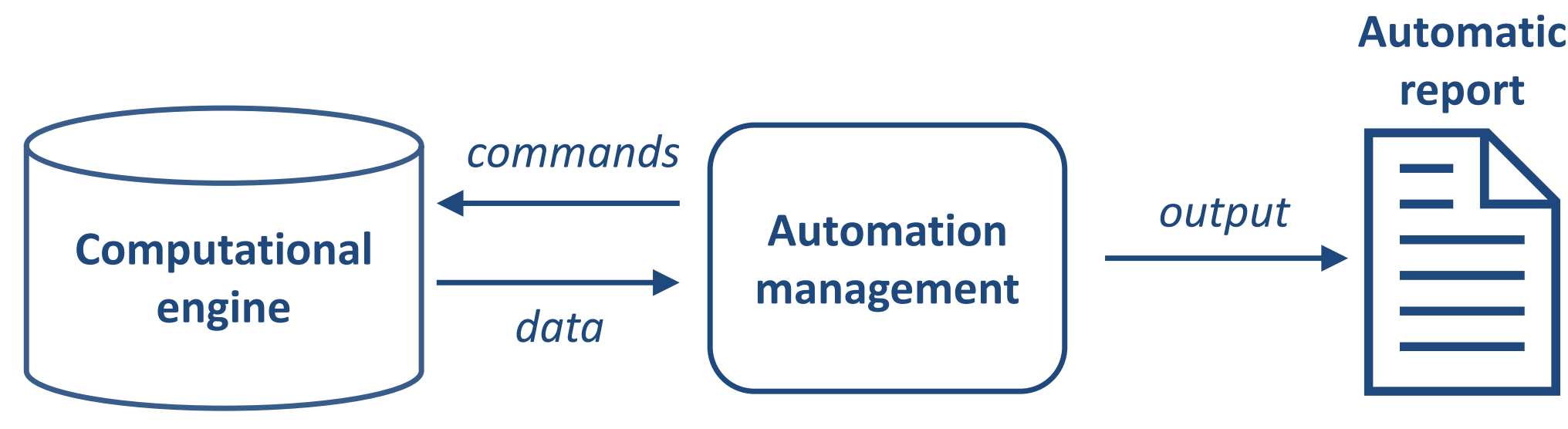
- 1 isttex by Newson (2003)

- textab by Hardin (1995)

- estout by Jahn (2005)

- estab by Jann (2007)

- outreg by Gallup (1998)

- Automatic generation of documents, discussed in Gini e Pasquini (2006)

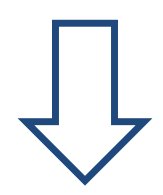

General limits:

- Tex/Latex oriented

- Not easy to learn

- Not "what you see is what you get approach" (WYSIWYG)

- Not complete solutions 


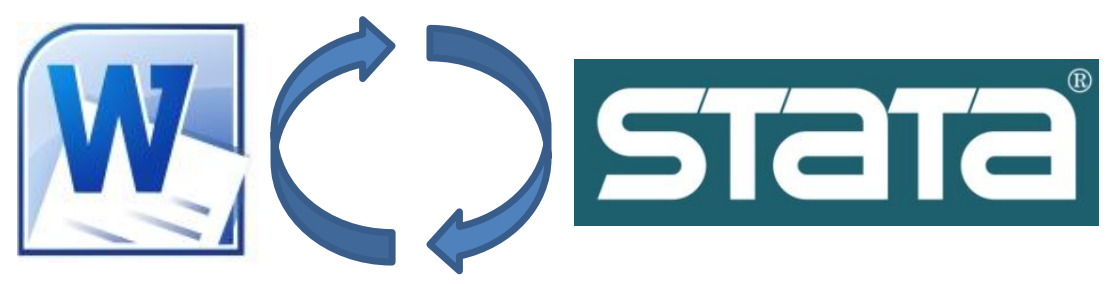

Sar is a software which allows you to automatically obtain numerical results from Stata in Word, making the formatting of statistical results easier

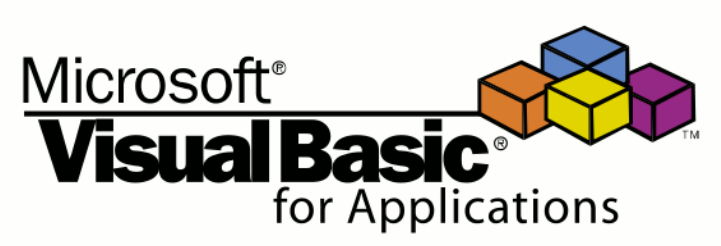

Sar is not a Stata command, but a macro for Microsoft Word written in the Visual Basic for Applications (VBA) programming language

Current version of Sar is 1.0 


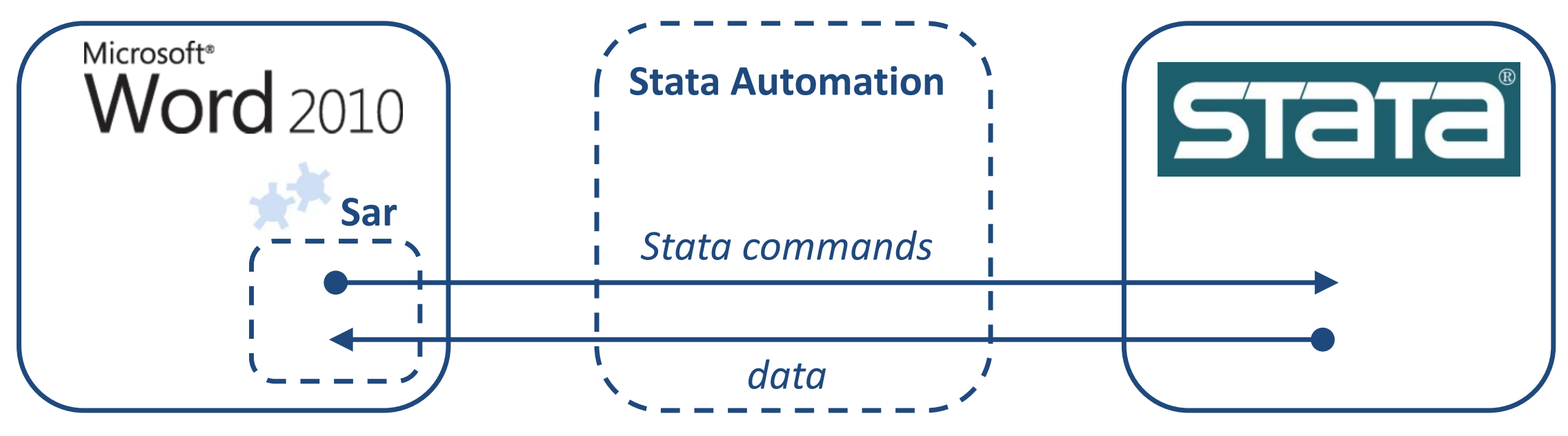

Stata Automation is a communication mechanism between Stata and Microsoft Windows applications (read www.stata.com/automation for details) 


\section{Before executing Sar}

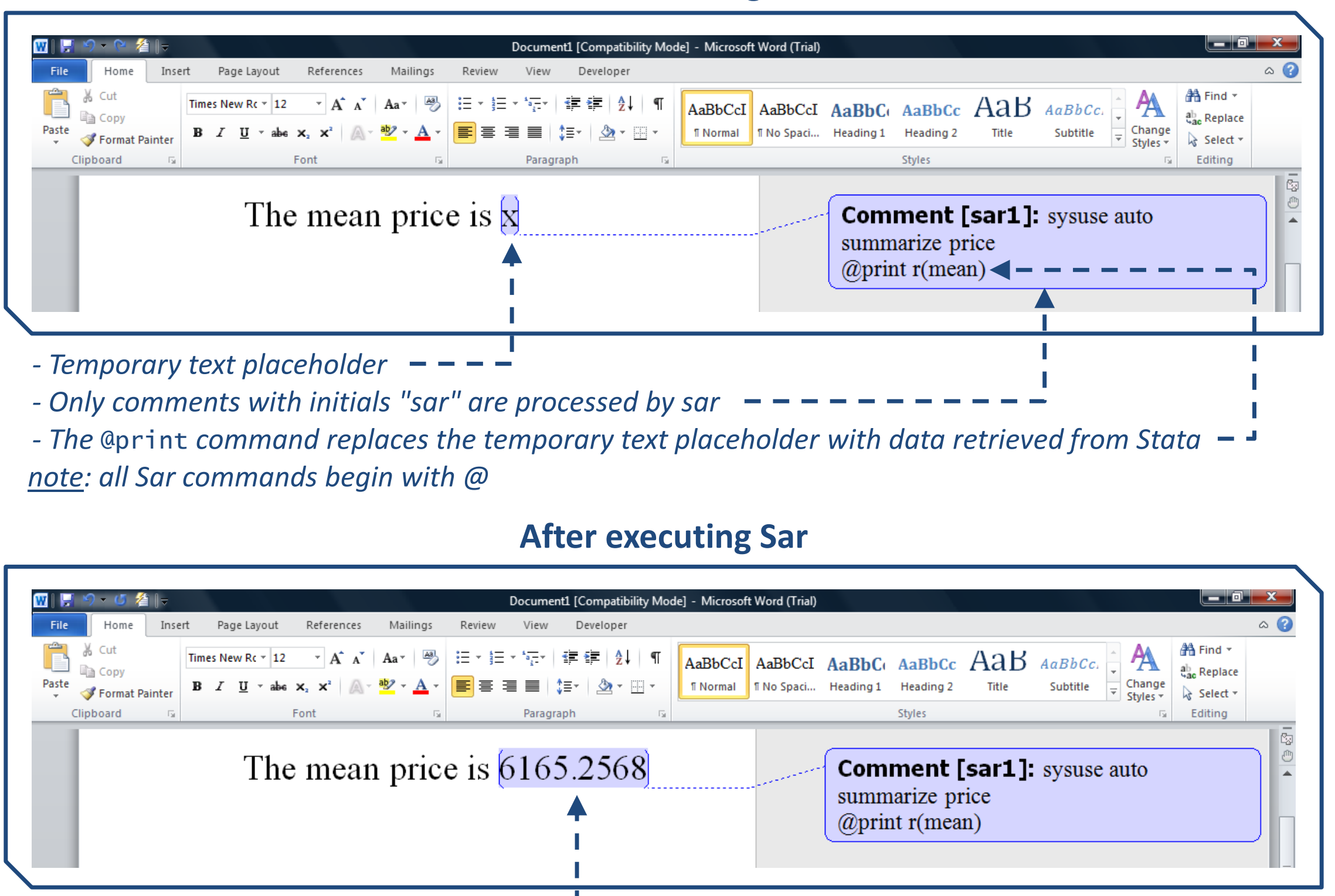

Data retrieved from Stata - - - - - - I 


\section{Wrong!}

\section{Comment [sar1]: use @: Imydata.dta?}

regress y $\mathrm{x}$

matrix beta $=\mathrm{e}(\mathrm{b}) \rho$

\section{Correct!}

\section{Comment [sar1]: use "C:Imydata.dta"} regress y $\mathrm{x}$ matrix beta $=\mathrm{e}(\mathrm{b})^{\prime}$ 


\section{$\square$ Software requirements}

- Microsoft Word for Windows (Stata Automation only runs on Windows)

- Stata (of course!)

\section{$\checkmark$ Manual settings}

- You have to install the Stata Automation object

- You have to copy the Sar macro ("Stata automatic report 1.0.dotm") in the Word startup folder

- Optionally, you can customize the Word quick access toolbar creating a button to easily execute the Sar macro

- You have to set user's initials of Word comments to "sar" 
1. Right-click on the Stata executable (on my machine the executable file is westata.exe and it is located in the $c:|S t a t a 10|$ folder)

2. Choose "Create shortcut" (I suggest you to rename the shortcut to "stata automation")

3. Right-click on the just created shortcut, choose "Property" and change Target from "C:|Stata10|westata.exe" to "C:|Stata10|westata.exe/Register" (please check the correcteness of your Stata path and its executable)

4. Right-click on the shortcut and choose "Run as administrator"

(read www.stata.com/automation for more details and informations about how to install the Stata Automation object on a Windows non-Vista machine) 


\section{Copying the Sar macro in the Word startup folder}

In Microsoft Word:

File $\Rightarrow$ Options $\Rightarrow$ Advanced $\Rightarrow$ File Locations...

\section{Word Options}

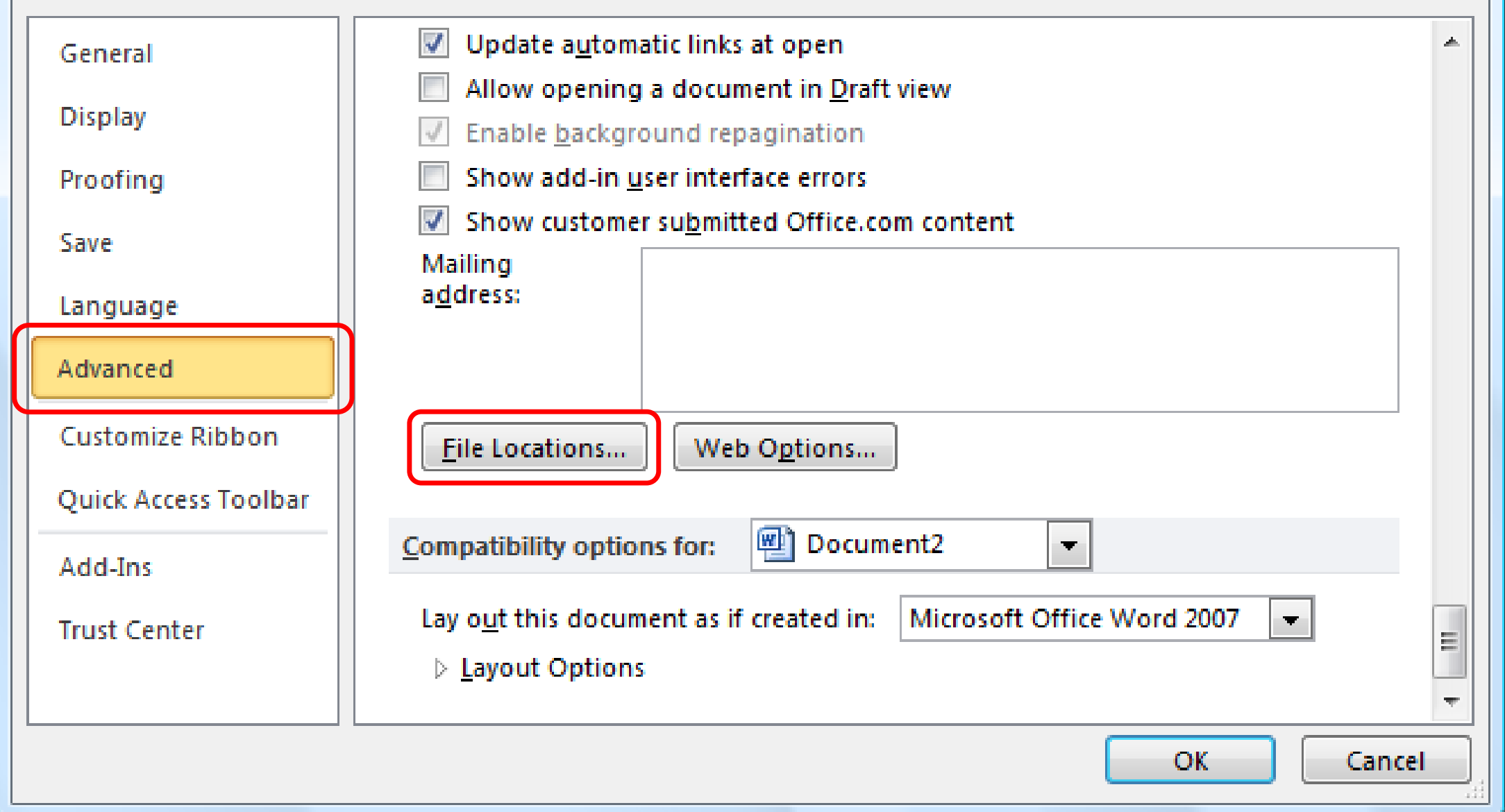

Click on the "File Locations..." button to find out where the Word startup folder is and copy the "Stata automatic report 1.0.dotm" macro file in it 
In Microsoft Word:

Customize Quick Access Toolbar $\Rightarrow$ More Commands...

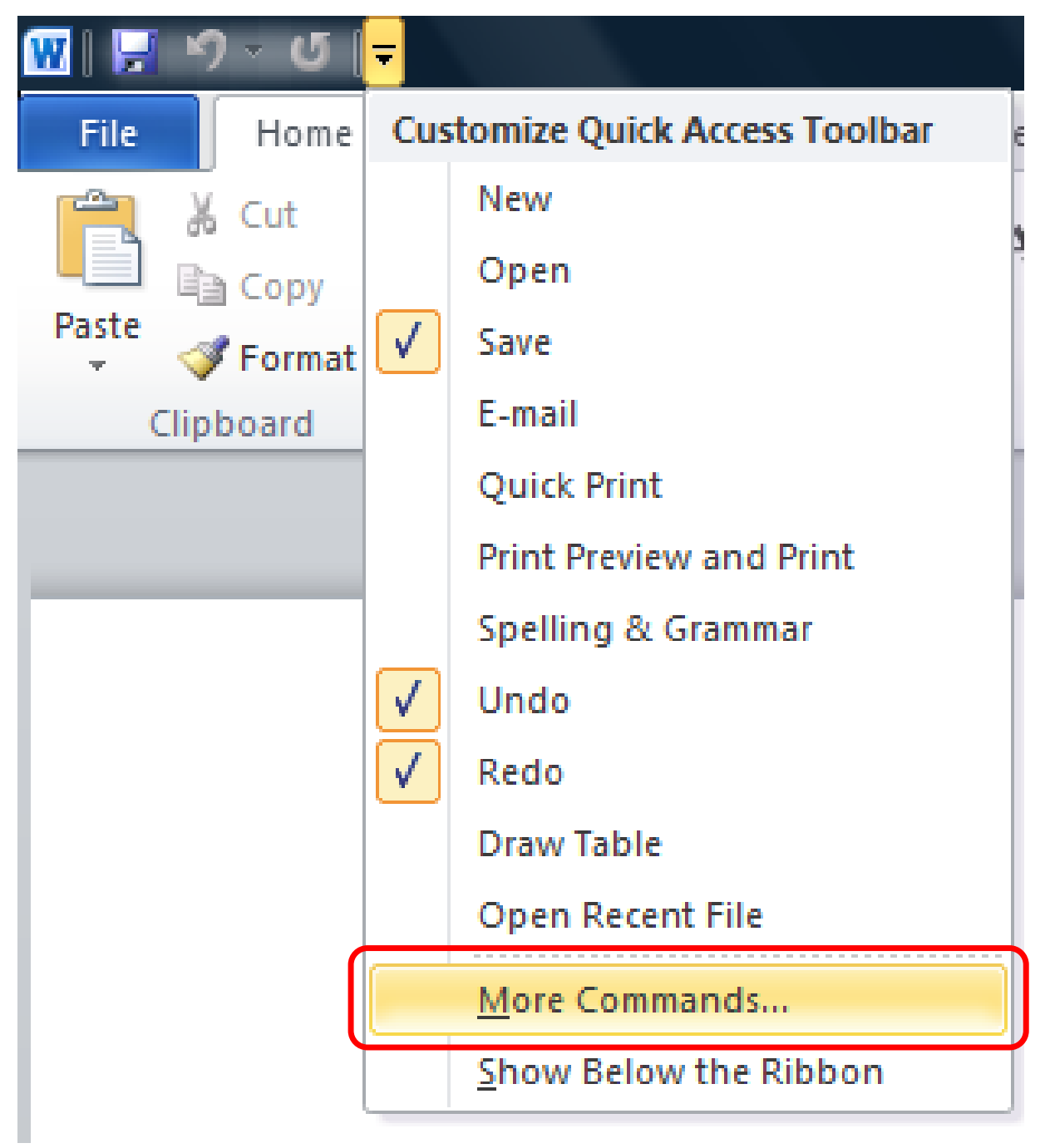




\section{Customize the Word quick access toolbar (Step 2 of 4)}

In Microsoft Word, after Step 1:

Choose "Macros" from the "Choose commands from" list $\Rightarrow$ Select the Stata automatic report macro $\Rightarrow$ Click on the "Add" button

Word Options

\begin{tabular}{|l|}
\hline General \\
Display \\
Proofing \\
Save \\
Language \\
Advanced \\
\hline Customize Ribbon \\
\hline Quick Access Toolbar \\
\hline Add-Ins \\
Trust Center \\
\hline
\end{tabular}

Customize the Quick Access Toolbar.
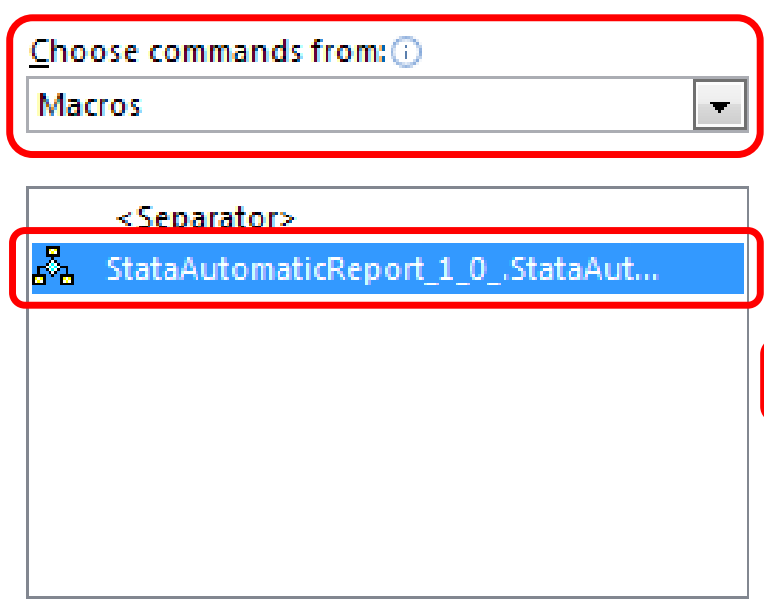

Show Quick Access Toolbar below the Ribbon

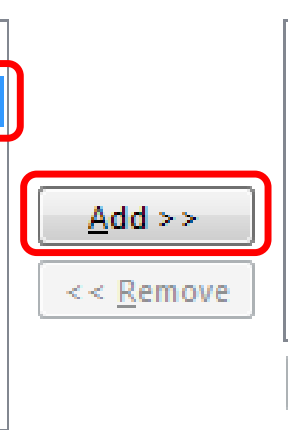

Modify...

Customizations:

\section{प. Save}

(9) Undo

$\checkmark$ Redo
Customize Quick Access Toolbar: (i)

For all documents (default)

\begin{tabular}{|c|c|}
\hline 8 & $x$ \\
\hline
\end{tabular}

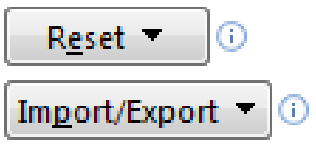




\section{Customize the Word quick access toolbar (Step 3 of 4)}

In Microsoft Word, after Step 2:

Select the Sar macro from the right list $\Rightarrow$ Click on the "Modify..." button to chooce an icon for the button which will be added to the quick access toolbar

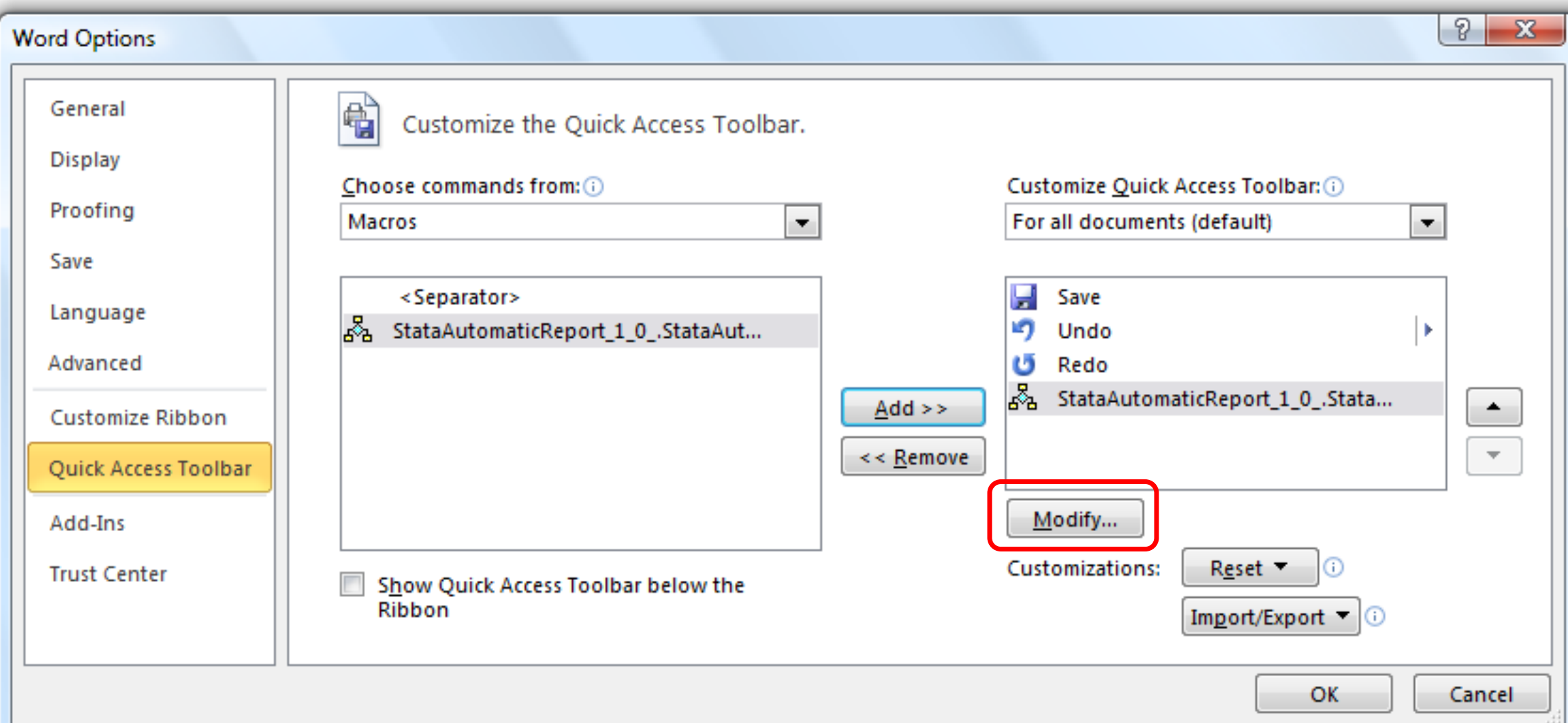


In Microsoft Word, after Step 3:

Choose your favorite icon and change the display name to "Stata automatic report 1.0"

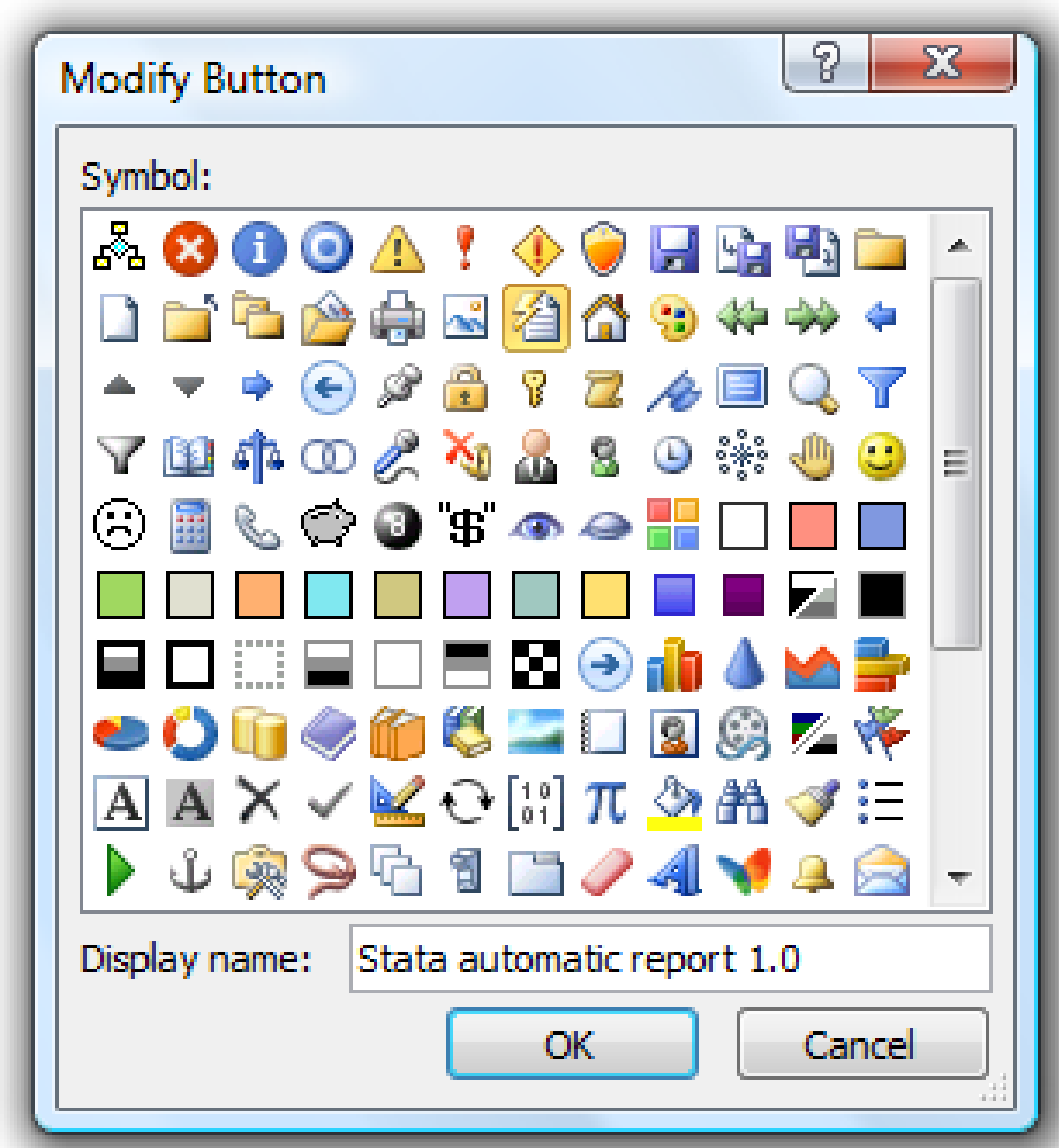

This is your Sar button on the quick access toolbar. You can launch the Sar macro by clicking on it

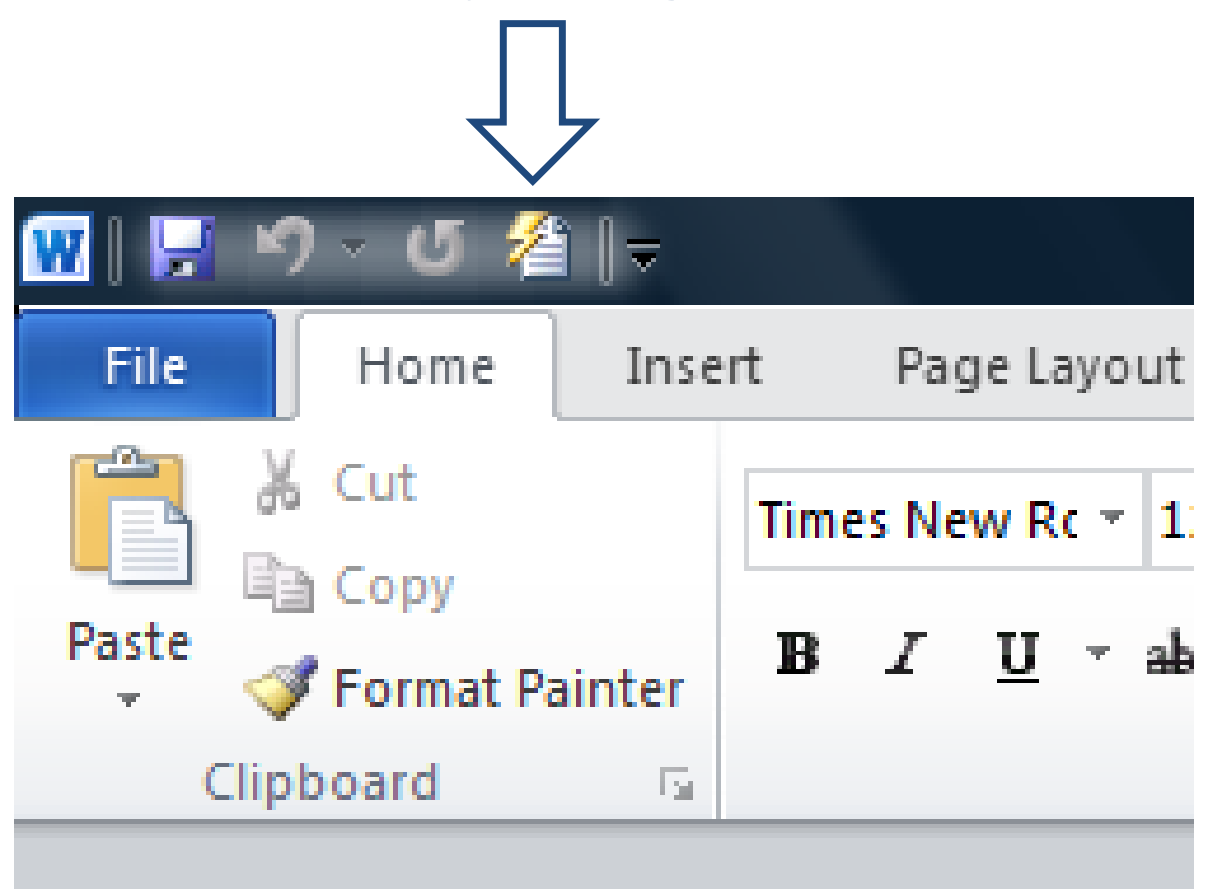


In Microsoft Word:

Word Options $\Rightarrow$ General $\Rightarrow$ Set user's initials to "sar"

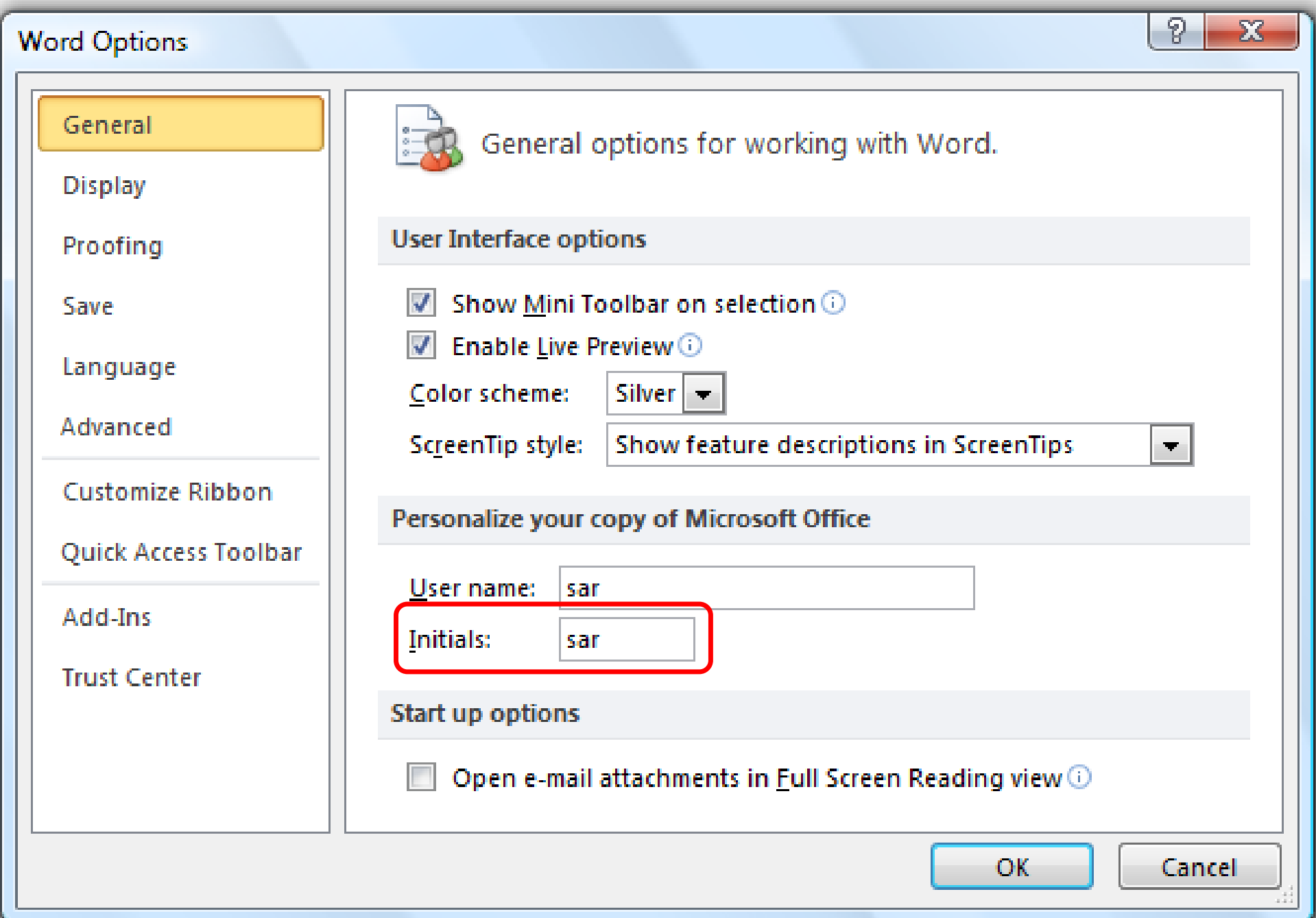




\author{
Syntax \\ aprint StataData \\ @format \%stataNumerica7Format
}

\title{
My beautiful report
}

Comment [sar1]: sysuse auto summarize price

In this report I comment some summary statistics from the auto.dta dataset.

The mean price is 6165.3 , the number of observations is 74 and there are 22 foreign cars.

Comment [sar2]: @format \%5.1f @print $\mathrm{r}$ (mean)

Comment [sar3]: @format \%2.0f @ print $\mathrm{r}(\mathrm{N})$

Comment [sar4]: count if foreign $==1$ @print $\mathrm{r}(\mathrm{N})$

Note: no leading spaces are added to the numerical output even if they are expected according to the Stata formatting rules 


\section{Syntax}

Qfil1table StataData startingRow startingco7 [rowstep co7step] Qmatrixrownames stataMatrix startingRow startingco 7 [rowstep]

\section{My regression analysis}

\begin{tabular}{lr}
\hline Variable & Coefficient \\
\hline weight & 4.7 \\
length & -98.0 \\
cons & 10386.5 \\
\hline
\end{tabular}

\section{Comment [sar1]: sysuse auto} regress price weight length matrix beta $=\mathrm{e}(\mathrm{b})^{\prime}$
Comment [sar2]: @format \%5.1f @filltable beta 22 @matrixrownames beta 21 


\section{Syntax}

Qfil1table StataData startingRow startingcol [rowstep co7step] @matrixrownames StataMatrix startingRow startingco 7 [rowstep]

abeginstring \#string\#

Qendstring \#string\#

\section{My regression analysis}

\begin{tabular}{lr}
\hline Variable & Coefficient \\
\hline weight & 4.7 \\
& $(1.1)$ \\
length & -98.0 \\
& $(39.2)$ \\
cons & 10386.5 \\
& $(4308.2)$ \\
\hline
\end{tabular}

Note: standard errors in parentheses
Comment [sar1]: sysuse auto regress price weight length matrix beta $=\mathrm{e}(\mathrm{b})^{\prime}$ mata: V = st_matrix("e(V)") mata: st_matrix("sd", sqrt(diagonal(V)))
Comment [sar2]: @format \%5.1f @filltable beta 2210 @beginstring \#(\# @endstring \#)\# @ filltable sd 3210 @matrixrownames beta 211 
Roughly speaking, a Sar program is a list of Sar and Stata commands:

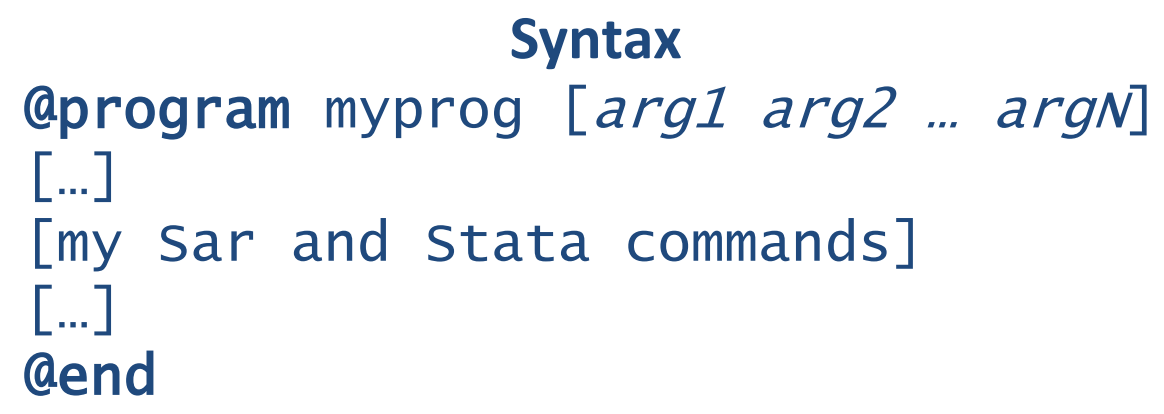

It can be defined:

- in a Word comment

- in a plain text file (called library in the Sar jargon)

It can be executed using the @do command:

\section{Syntax}

@do myprog [ [arg1 arg2 ... 


\section{Program definition}

\begin{tabular}{|c|c|c|}
\hline \multirow{5}{*}{ program name } & aprogram outmatrixmatrix & \multirow{5}{*}{$\begin{array}{l}\text { argument } \\
\text { callbacks }\end{array}$} \\
\hline & @matrixrownames §matrixई 21 & \\
\hline & 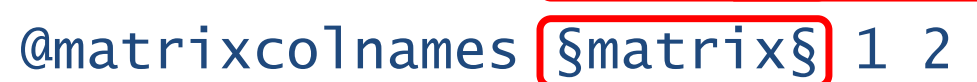 & \\
\hline & aformat $\% 4.3 f$ & \\
\hline & afi11table §matrix\$ 22 & \\
\hline
\end{tabular}

\section{Example of usage}

correlate price weight length ado outmatrix r(c)

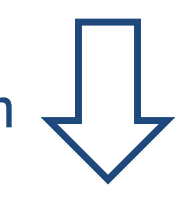

The compiled program

aprogram outmatrix $r(C)$

@matrixrownames $r$ (C) 21

@matrixcolnames $r(C) 12$

@format \%4.3f

afil1table r(c) 22

aend 
Comment [sar1]: @program outmatrix matrix

@matrixrownames §matrix $\S 21$

$@$ matrixcolnames §matrix $\S 12$

@ format $\% 4.3 \mathrm{f}$

$@$ filltable §matrix $\S 22$

@end

Comment [sar2]: sysuse auto correlate price weight length @ do outmatrix r(C)

You can use the just defined outmatrix program how many times you want in your Word document 


\section{Syntax \\ @1oad1ibrary "pathofTheLibraryfi7e"}

Plain text file: c:|sar libraries|mylibrary.txt

aprogram outmatrix matrix

@matrixrownames §matrix§ 21

@matrixcolnames §matrix§ 12

aformat \%4.3f

afil1table §matrix§ 22

Qend

\section{A correlation matrix}

Comment [sar1]: @loadlibrary "c:lsar libraries $\backslash$ mylibrary.txt"

\begin{tabular}{rrrr}
\hline & price & weight & length \\
\hline price & 1.000 & 0.539 & 0.432 \\
weight & 0.539 & 1.000 & 0.946 \\
length & 0.432 & 0.946 & 1.000 \\
\hline
\end{tabular}

Comment [sar2]: sysuse auto correlate price weight length @do outmatrix r(C) 


\section{Syntax}

Qresetstring

(no arguments are required)

Plain text file: c:|sar libraries|mylibrary.txt

\begin{tabular}{|c|c|}
\hline 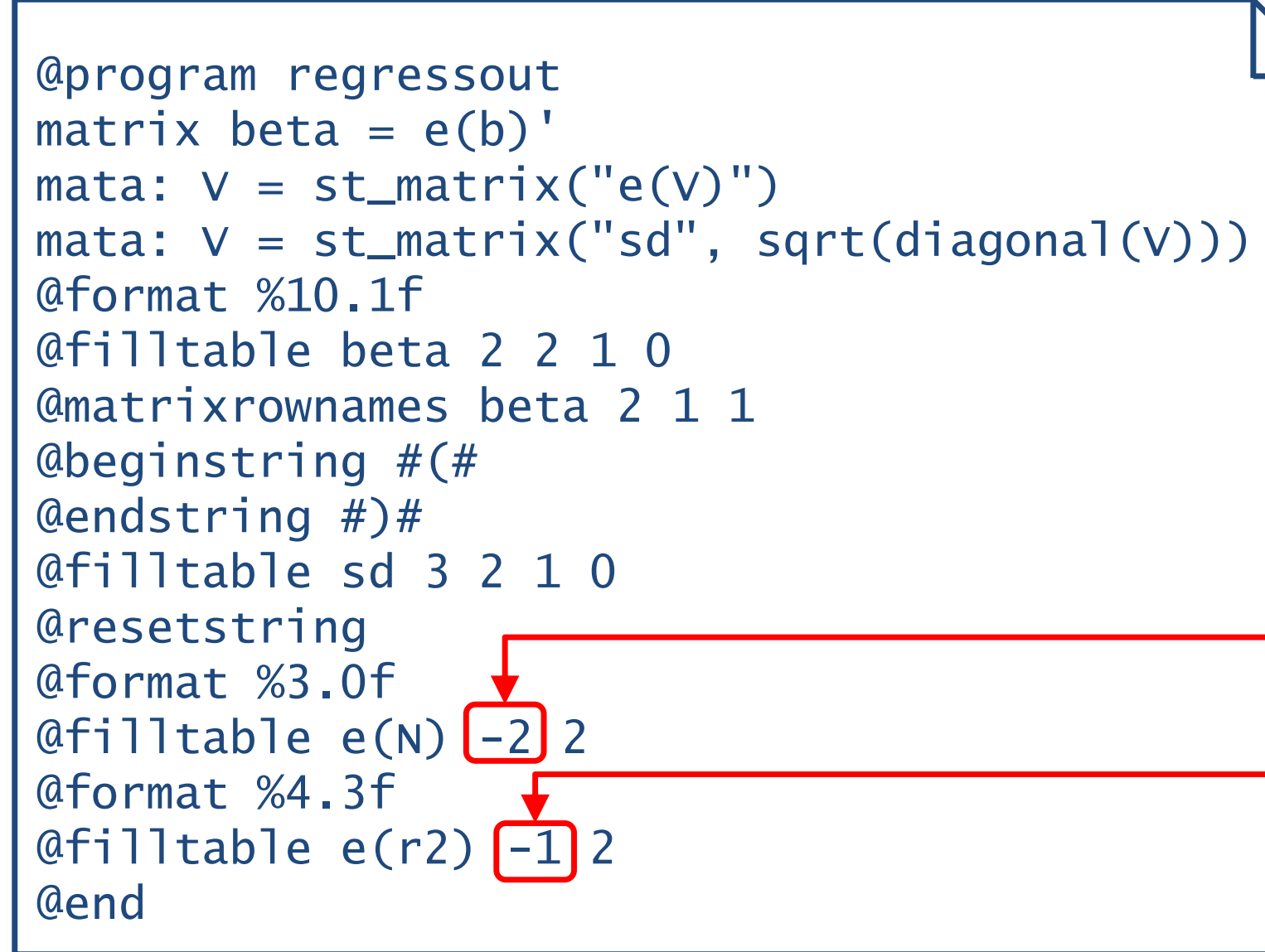 & $\begin{array}{l}\text { When arguments of the } \\
\text { @filltable command are }-1 \text {, } \\
-2 \text {, etc... they indicate the } \\
\text { last row/column, the } \\
\text { second last row/column } \\
\text { and so on. }\end{array}$ \\
\hline
\end{tabular}


Comment [sar1]: sysuse auto

@loadlibrary "c:Isar libraries\mylibrary.txt"

\begin{tabular}{lr}
\hline Variable & Coefficient \\
\hline weight & 4.7 \\
length & $(1.1)$ \\
& -98.0 \\
cons & $(39.2)$ \\
& 10386.5 \\
$N$. of obs. & $(4308.2)$ \\
$R^{2}$ & 74 \\
\hline Note: standard errors in parentheses
\end{tabular}

Comment [sar2]: regress price weight length

@do regressout

Note: Sar is not verbose!

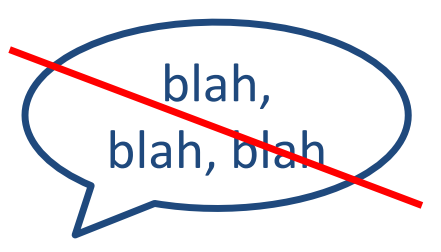




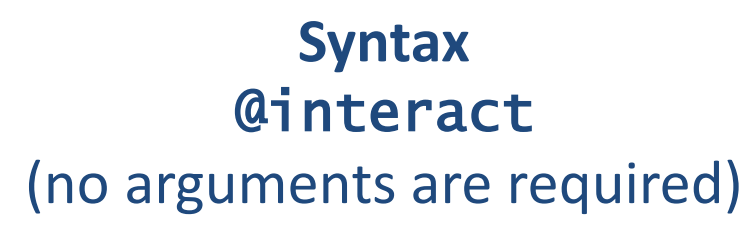

Example: our goal is to create the well-known $\left(X^{\prime} X\right)^{-1}$ matrix

The execution of Sar will halt here, allowing the user to interact with Stata

\section{A tricky matrix}

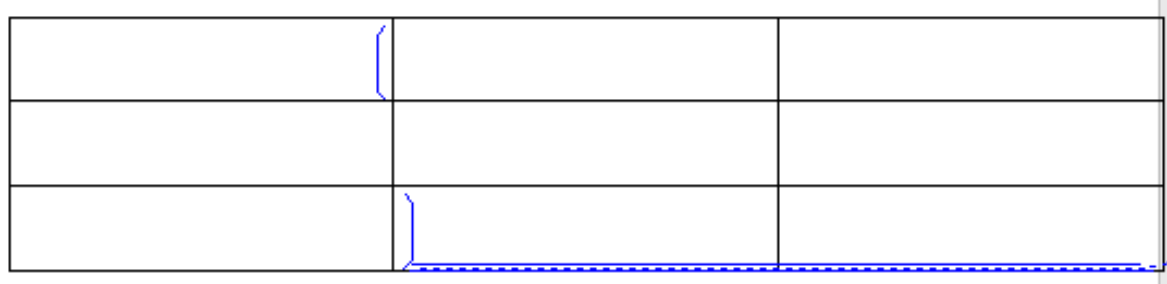

Comment [sar1]: @interact @filltable mymatrix 11

This matrix will be created

by the user in Stata 


\section{Using Sar in interactive mode (Step 2 of 3 )}

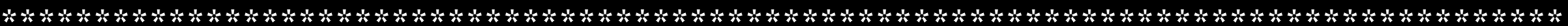

* suppose you don't remember how to use the mkmat command

- help mkmat

- mkmat mpg weight, matrix(X)

- count

74

- $\operatorname{matrix}$ one $=\mathrm{J}(74,1,1)$

- matrix $X=X$, one

- matrix mymatrix $=\operatorname{invsym}\left(X^{\prime} * X\right)$
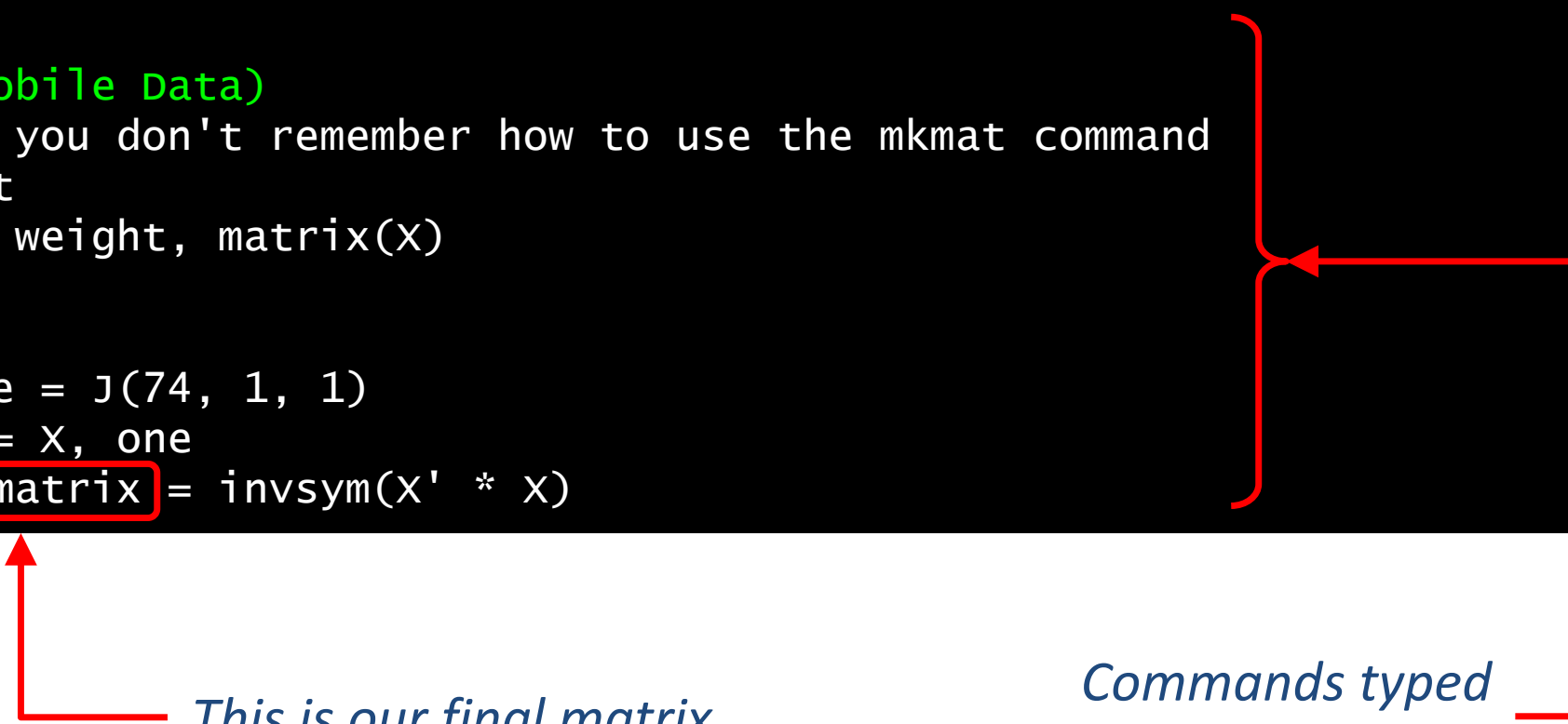

This is our final matrix

Commands typed by the user in Stata

This Stata window has to be closed from Word (see Step 3) 
This dialog window will be opened in Word after the execution of @interact

Don't forget to click here after interacting with Stata!

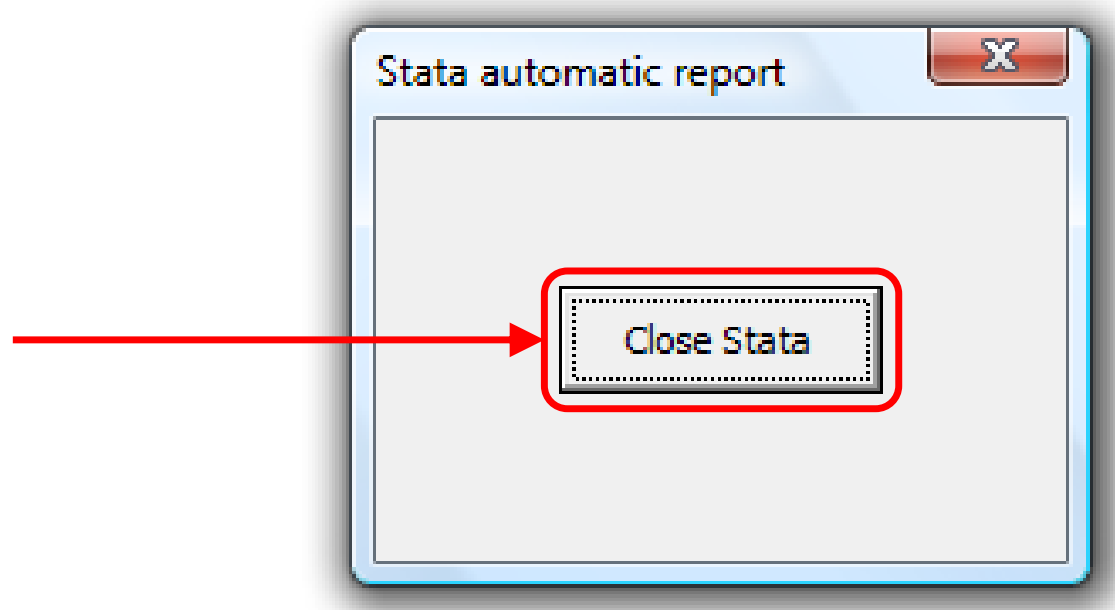

This is the final output:

\section{A tricky matrix}

\begin{tabular}{|r|r|r|}
\hline .00117444 & $7.057 \mathrm{e}-06$ & -.04632027 \\
\hline $7.057 \mathrm{e}-06$ & $6.508 \mathrm{e}-08$ & -.0003468 \\
\hline-.04632027 & -.0003468 & $2.047163)$ \\
\hline
\end{tabular}

Comment [sar1]: @interact $@$ filltable mymatrix 11

Probably you will delete this Sar comment (it's useless) 
Do file: c:|mydofile.do

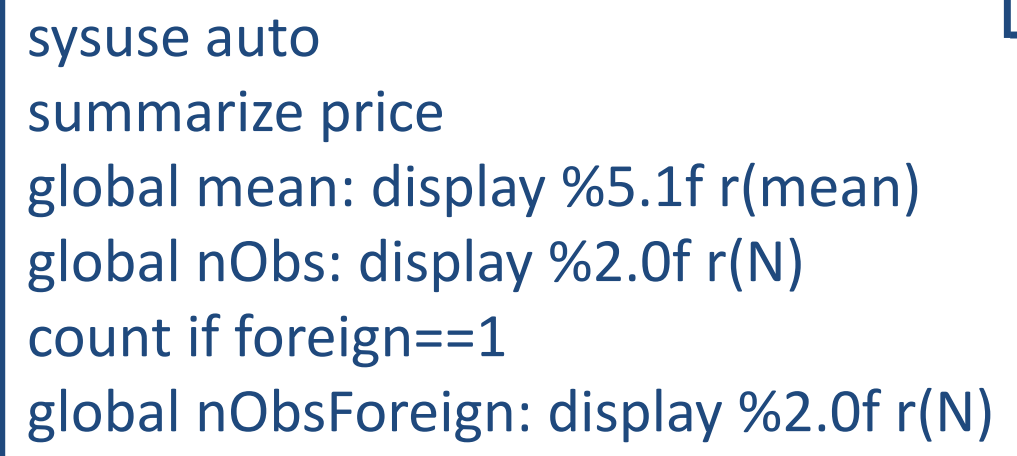

\section{My beautiful report}

Comment [sar1]: do "c:Imydofile.do"

In this report I comment some summary statistics from the auto.dta dataset.

The mean price is 6165.3 , the number of observations is 74 and Comment [sar2]: @print mean there are 22 foreign cars.

Comment [sar3]: @print nObs

Comment [sar4]: @print nObsForeign 
Creating automatic reports

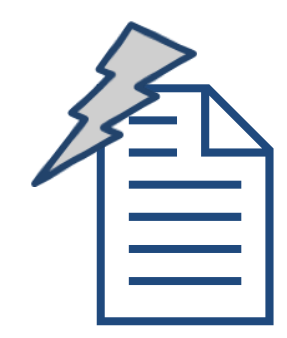

- There is no need to edit your report if data have changed

- The report is well documented
Using Sar in interactive mode

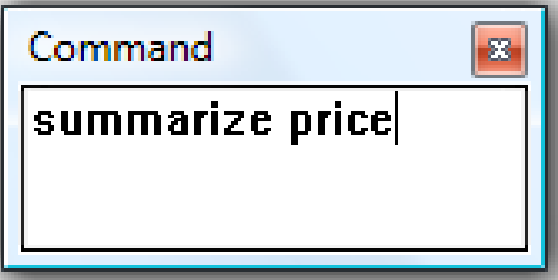

You can obtain data from Stata on the fly
Calling do files from Sar

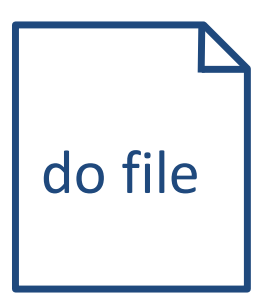

- You can test your do file in Stata

- You can store your statistical analysis in a do file 
- Automatic reports: documents which can auto-update themselves if data have changed

- Self-explaining data analysis

- WYSIWYG approach exploiting all the functions of Word

- Ease of learning

- Only 15 keywords

- Sar documents are not verbose

- Extensibility through Sar programs 
- Sar only works in Windows

- Lack of "undo" function to erase all changes made by Sar on the document

- The following Stata commands can not be used: program define, whi 1e, forvalues, foreach and input (but they can be used in do files)

- The @print command can not be used inside a Word table

- Setting of global and local macros has no effect in Sar

- Word comments with Sar commands can not refer to the same portion of a text

- You have to avoid to use the macro names "stataAutomaticReportValue" and "stataAutomaticReportMatrix", because they are interally used by Sar 


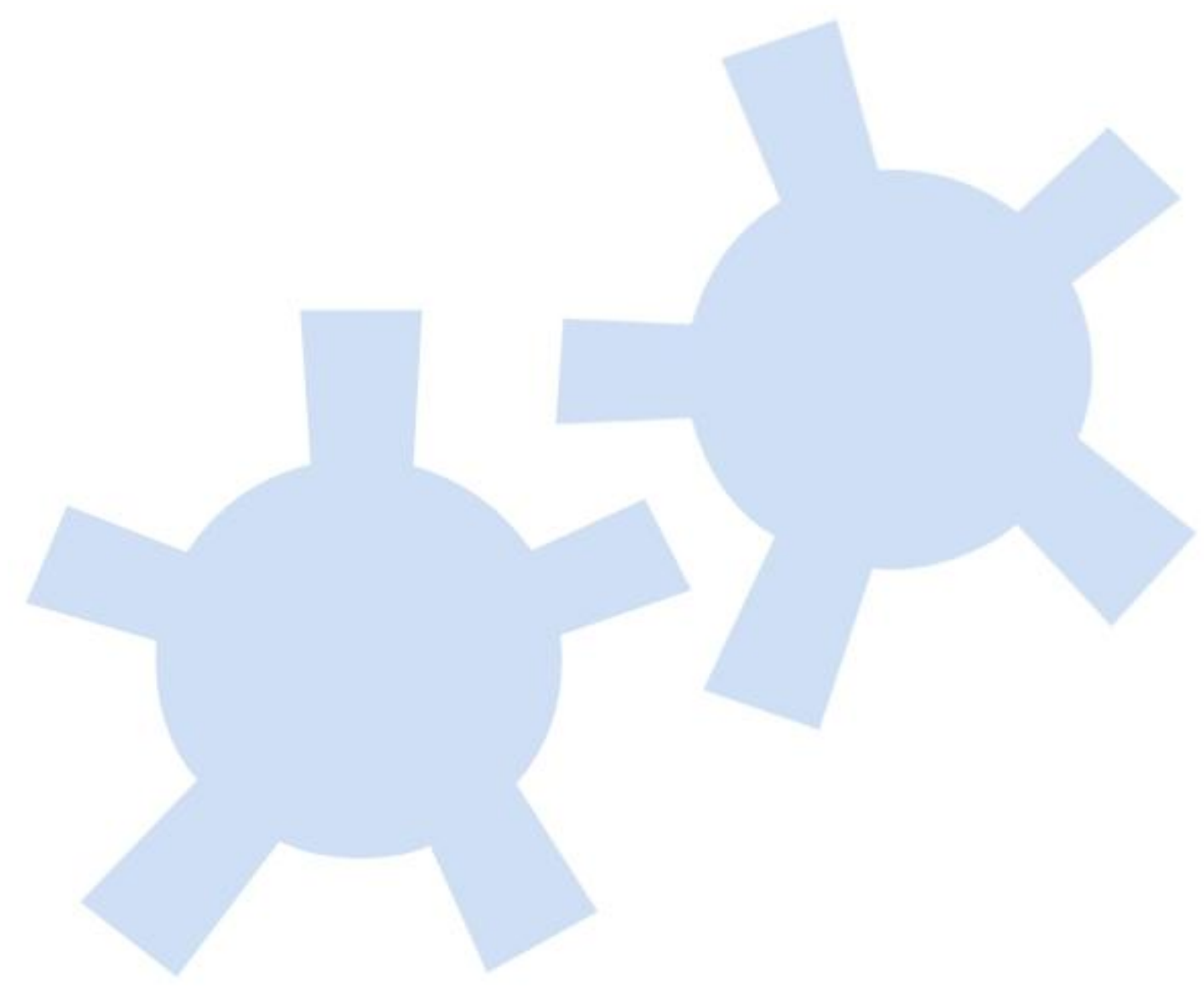

Thank you for your attention 


\section{The problem}

Students like copying from their schoolmates during statistics classwork

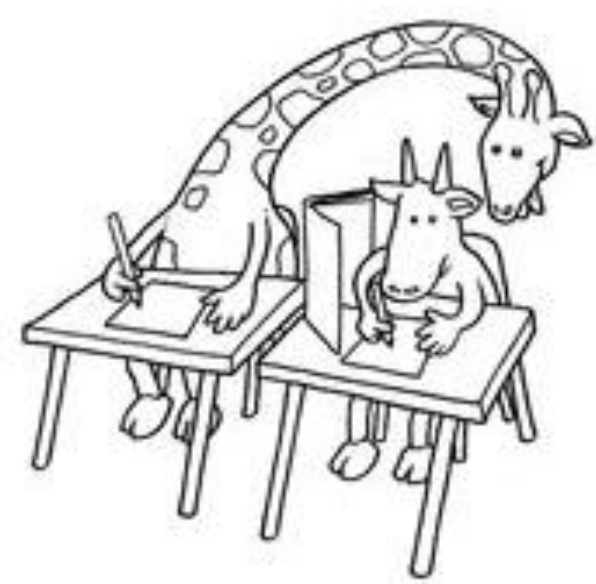

The solution

An automatic document created with Sar where numerical values of the exercises are randomly sampled from a dataset. A code-seed is uniquely assigned to each student. The teacher can use the code-seed to quickly reproduce the sampled dataset and mark the schoolwork. 


\section{Appendix A - Automatic classwork}

\section{Schoolwork}

Student's name:

Student's ID:21

\section{Exercise 1}

Calculate the Pearson correlation coefficient between the price and the weight of the following 7 cars:

\begin{tabular}{lrr}
\hline Make & Price & Weight \\
\hline VW_Diesel & 5397 & 2040 \\
Buick_Skylark & 4082 & 3400 \\
Merc_XR-7 & 6303 & 4130 \\
Toyota_Corona & 5719 & 2670 \\
Buick_Century & 4816 & 3250 \\
Subaru & 3798 & 2050 \\
Chev_Malibu & 4504 & 3180 \\
\hline
\end{tabular}

Comment [sar1]: sysuse auto

set seed 21

sample 7 , count

encode make, generate(makeNumeric)

mkmat makeNumeric, matrix(make)

rownames(make)

mkmat price, matrix (price)

mkmat weight, matrix(weight)
You should manually change the seed in the comment and in the document, launch Sar and print the document: that's very boring if you have many students. It's better to use the mail-merge functions by Word.
Comment [sar2]: @matrixrownames make 21

@filltable price 22

@ filltable weight 23 


\section{What is mail-merge is?}

It is a software function which allows you to create multiple documents from a template

How you can access the mail-merge functions of Word:

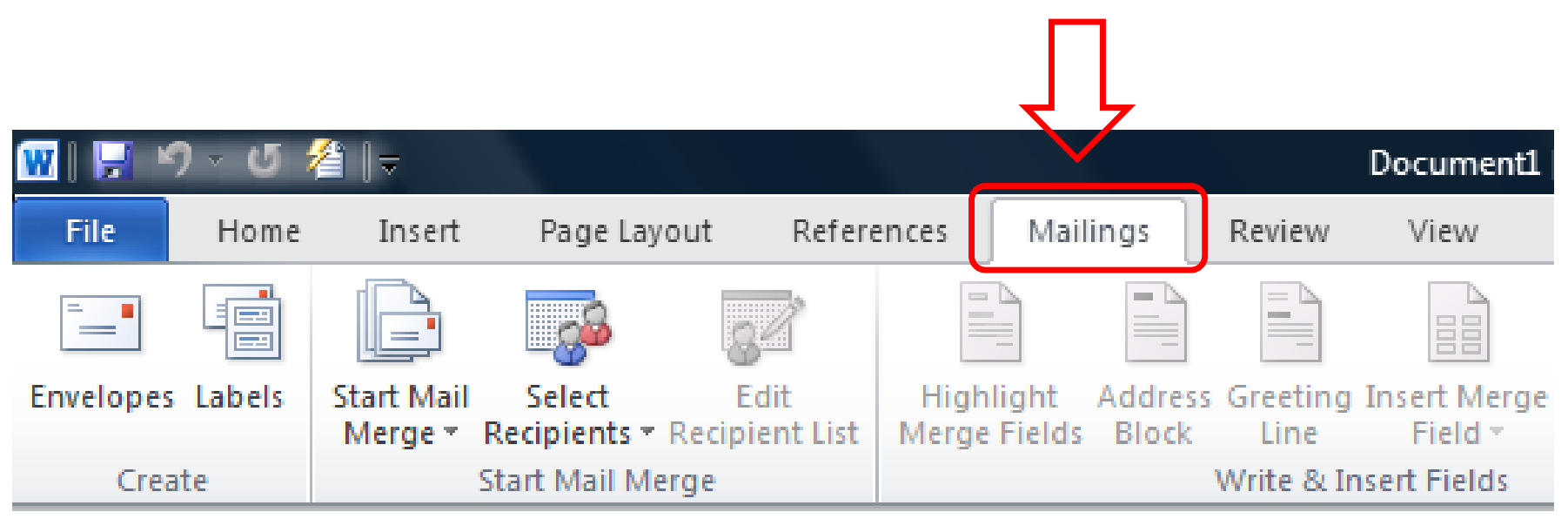


Step 1: Select the "letters» document type

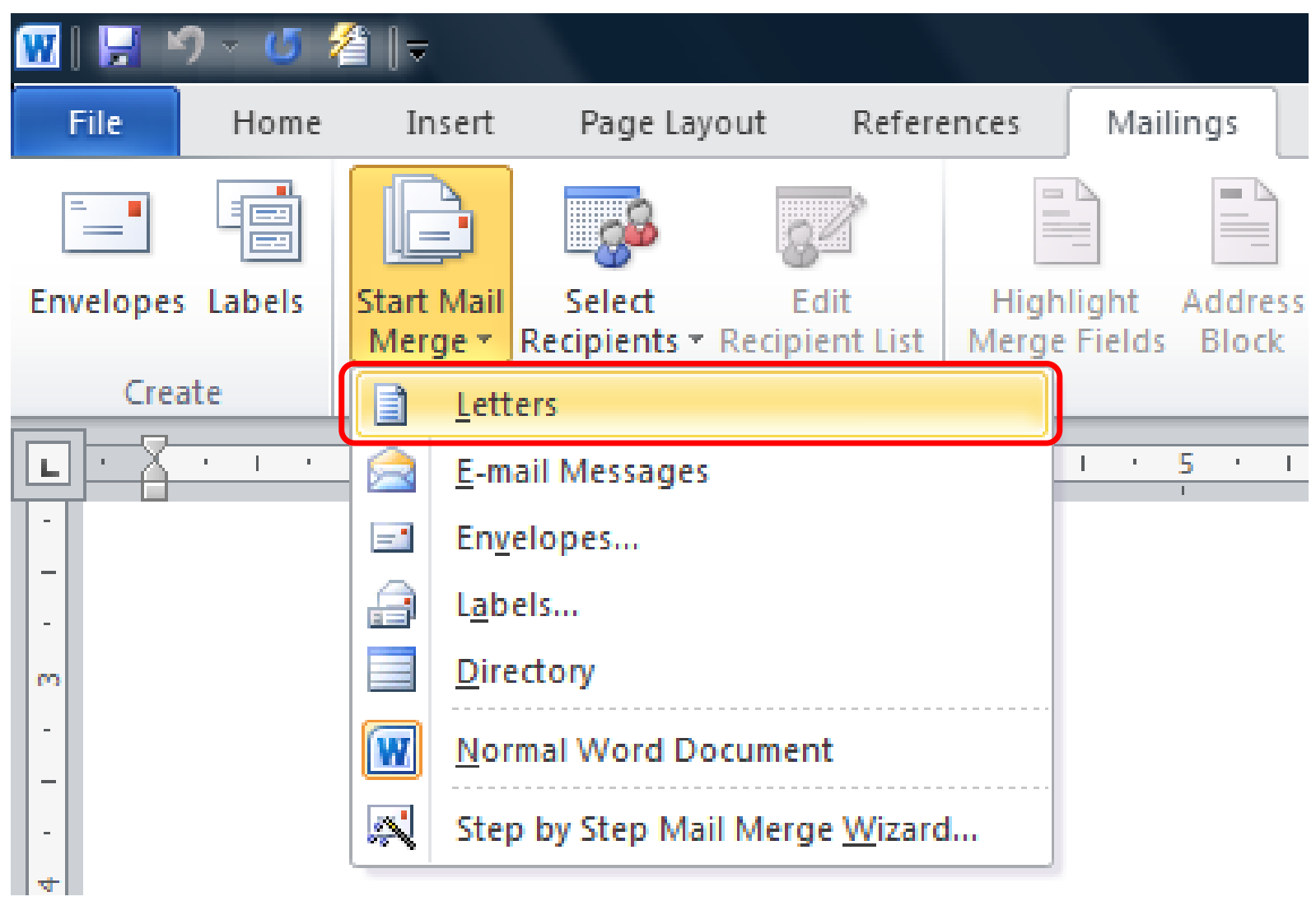


Step 2: Create a new database

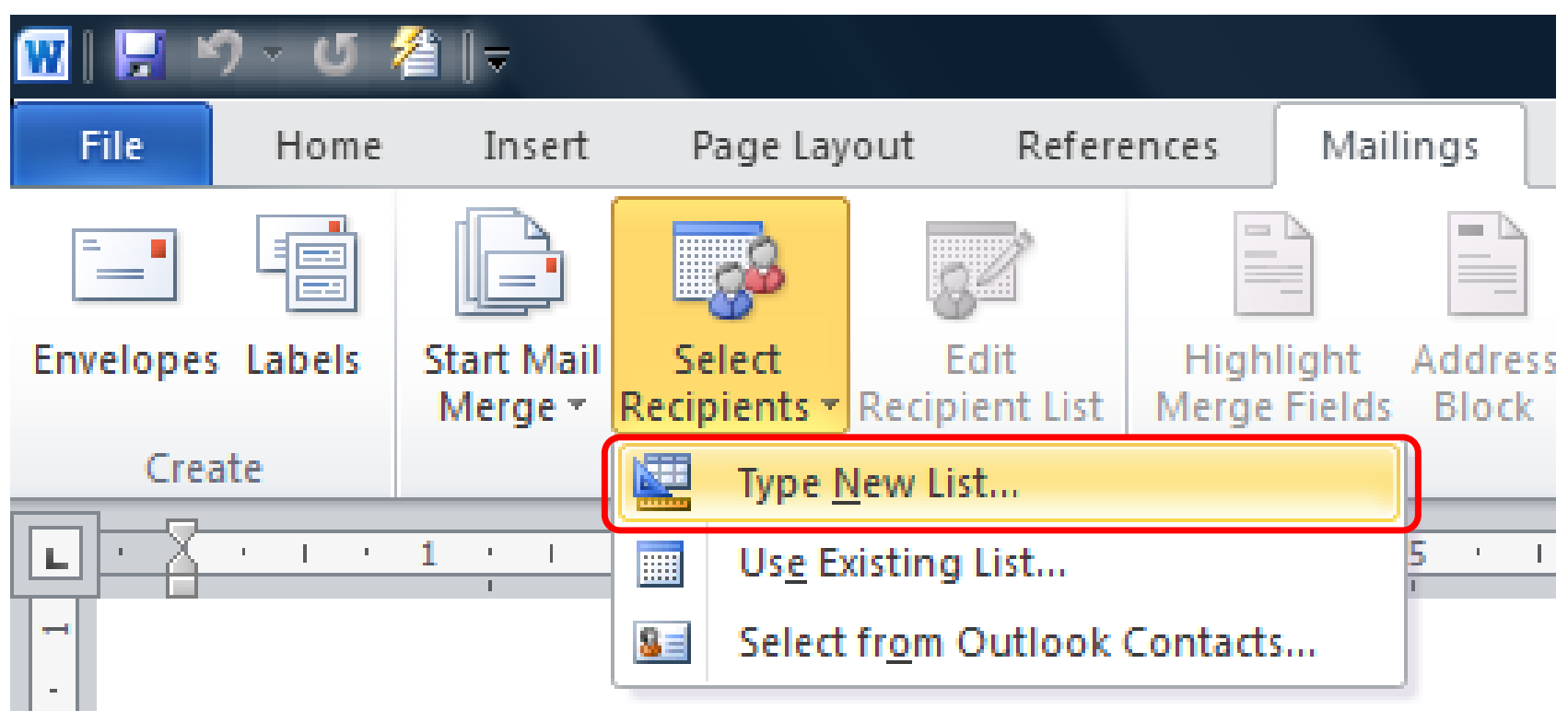


Step 3: Fill the database with your students' names and a univocal ID

\begin{tabular}{|c|c|c|c|c|c|c|c|c|}
\hline \multicolumn{6}{|c|}{ New Address List } & \multicolumn{3}{|c|}{\begin{tabular}{|l|l|}
8 & $x$ \\
\end{tabular}} \\
\hline \multicolumn{9}{|c|}{ Iype recipient information in the table. To add more entries, click New Entry. } \\
\hline \begin{tabular}{|l|l|l|l|} 
Title \\
\end{tabular} & $\boldsymbol{\nabla}$ & ID $\quad \boldsymbol{\nabla}$ & First Name & $\boldsymbol{\nabla}$ & Last Name & $\nabla$ & Company Name $\boldsymbol{\nabla}$ & $\Delta$ \\
\hline & & 1 & Christine & & Giraffe & & & \\
\hline & & 2 & John & & Goat & & & \\
\hline & & 3 & Carmen & & Donkey & & & $\equiv$ \\
\hline & & 4 & Vincent & & Bird & & & \\
\hline | & & 5 & Andrew & & Hedgehog & & & L \\
\hline & & 6 & Francis & & Galton & & & \\
\hline 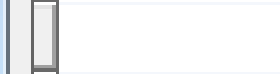 & & 7 & Andrej & & Kolmogorov & & & \\
\hline 7 & & 8 & William & & Cochran & & & \\
\hline 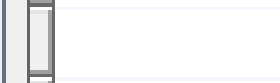 & & 9 & Abraham & & Wald & & & \\
\hline$\pi$ & & 10 & David & & Kendall & & & + \\
\hline $1 \square$ & \multicolumn{2}{|c|}{ III } & & & & & \multicolumn{2}{|l|}{+} \\
\hline New Entry & \multicolumn{2}{|r|}{ Find... } & & & & & & \\
\hline Delete Entry & \multicolumn{2}{|c|}{ Customize Columns... } & & & & OK & \multicolumn{2}{|l|}{ Cancel } \\
\hline
\end{tabular}

Click here to add the «ID» column 


\section{Appendix A - Automatic classwork}

Step 4: Create the template

\section{Schoolwork}

Student's name:

Student's ID:

\section{Exercise 1}

Calculate the Pearson correlation coefficient between the price and the weight of the following 7 cars:

\begin{tabular}{|l|l|l|}
\hline Make & Price & Weight \\
\hline & & \\
\hline & & \\
\hline & & \\
\hline & & \\
\hline & & \\
\hline & & \\
\hline
\end{tabular}

Comment [sar1]: sysuse auto, clear set seed

sample 7, count

encode make, generate(makeNumeric)

mkmat makeNumeric, matrix (make) rownames(make)

mkmat price, matrix (price)

mkmat weight, matrix(weight)

Don't worry about this

incomplete command (seed number is missing)

Don't forget to use the "clear» option

Comment [sar2]: @matrixrownames make 21

(a) filltable price 22

@ filltable weight 23 
Step 5: Insert merge fields

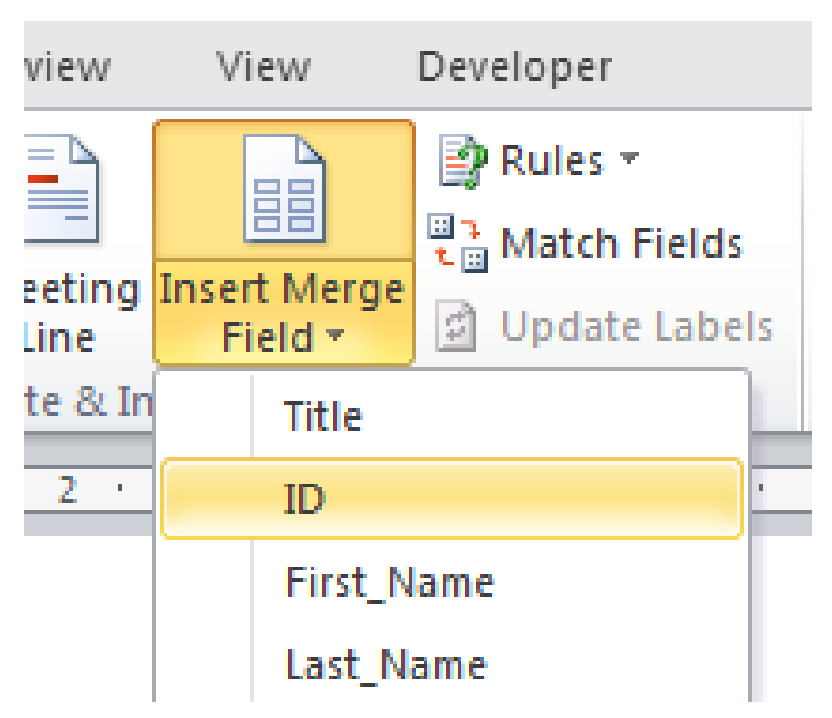

\section{(Schoolwork)}

Comment [sar1]: sysuse auto, clear set seed 《ID» sample 7, count encode make, generate(makeNumeric) mkmat makeNumeric, matrix(make) rownames(make) mkmat price, matrix (price) mkmat weight, matrix(weight)
Student's name: «First_Name» «Last_Name》 Student's D: $\lll \mathrm{D} \gg$ 
Step 6: Preview results

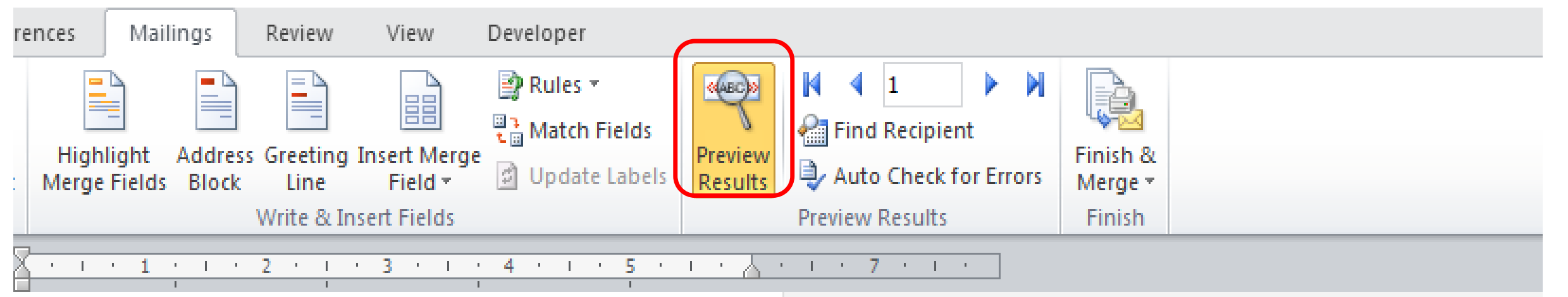

\section{Schoolwork}

Comment [sar1]: sysuse auto, clear set seed 1 sample 7, count encode make, generate(makeNumeric) mkmat makeNumeric, matrix(make) rownames(make) mkmat price, matrix(price) mkmat weight, matrix(weight)

\section{Exercise 1}

Calculate the Pearson correlation coefficient between the price and 


\section{Appendix A - Automatic classwork}

Step 7: Add a page break at the end of the document

\section{Schoolwork}

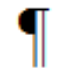

Student's name: Christine Giraffe

Student's.ID: 19

ฯ

\section{Exercise $\cdot 19$}

Calculate the Pearson - correlation . coefficient between the price and the weight $\cdot$ of the following $\cdot 7 \cdot$ cars: 9

\begin{tabular}{|c|c|c|}
\hline Maked & Price & Weight \\
\hline a & & o \\
\hline a & & o \\
\hline a & & c \\
\hline a & & c \\
\hline a & & c \\
\hline a & & c \\
\hline a & & $\phi$ \\
\hline
\end{tabular}

Comment [sar1]: sysuseauto, clear set $\cdot$ seed $\cdot 1$ sample 7 , count 1 encode 'make, generate(makeNumeric) mkmat makeNumeric, matrix (make). rownames(make) mkmat price, matrix (price) mkmat weight, matrix(weight)
Comment [sar2]: @matrixrownames. make $2 \cdot 19$ @ filltable.price.2.29 @filltable·weight·2·3 
Step 8: Merge to a new document
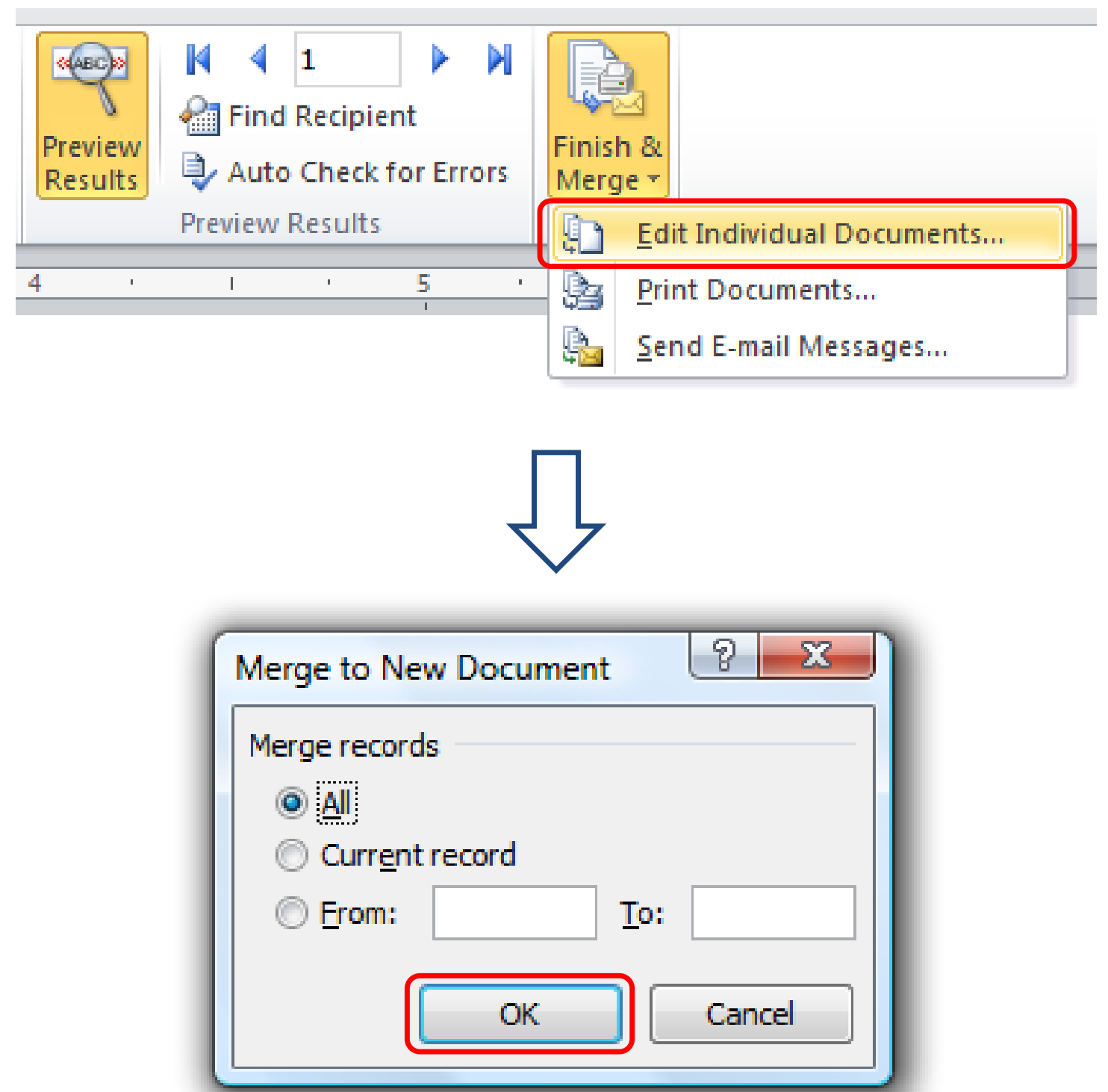
Problem: an artifact is generated by Word in the «mail-merge» document

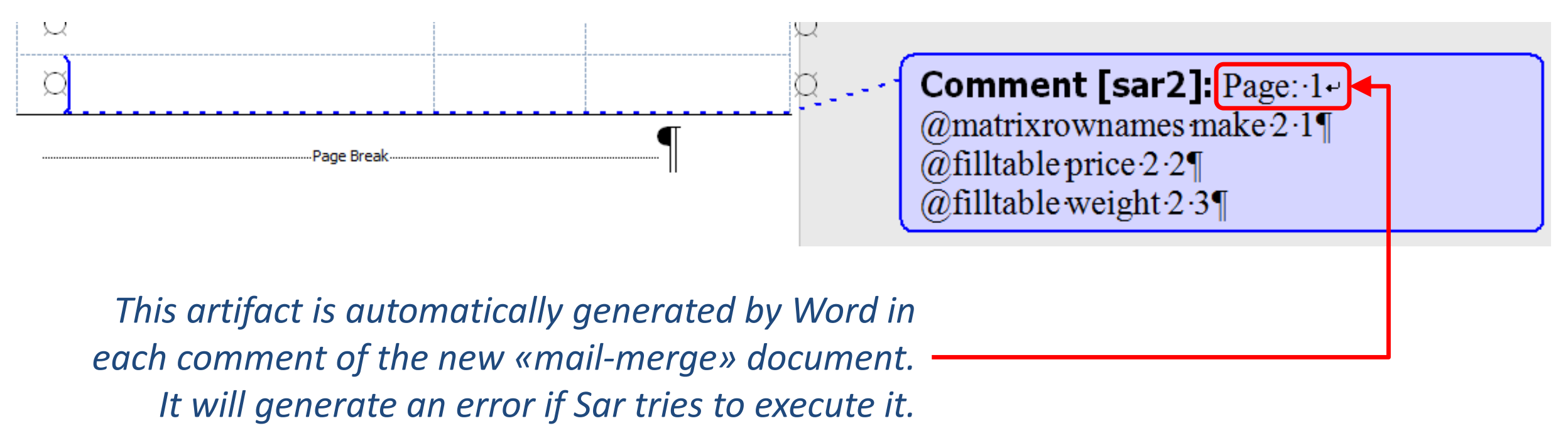


Step 9: Correct the generated by Word artifact in the «mail-merge» document

Find and Replace

\begin{tabular}{|l|l|l|l|l}
\hline 8 & 23 \\
\hline
\end{tabular}

Find Replace Go To

Find what: Page: 1 시

Replace with:

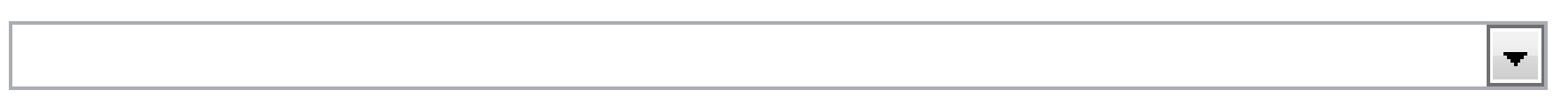

More $>>$

Replace

Replace ㅅl Find Next

Cancel

Notes:

- The string you have to replace is "Page: $1 \wedge / »$, where "^/» is a special character for "manual line break»

- Leave the "replace with» field void 
Step 10: Launch Sar from the «mail-merge» document and print the schoolwork for your students

\section{Schoolwork}

Student's name: Christine Giraffe

Student's ID: 1

\section{Excercise 1}

Calculate the Pearson correlation coefficient between the price and the weight of the following 7 cars:

\begin{tabular}{lrr}
\hline Make & Price & Weight \\
\hline Dodge_Colt & 3984 & 2120 \\
Chev_Nova & 3955 & 3430 \\
Chev_Chevette & 3299 & 2110 \\
Linc_Mark_V & 13594 & 4720 \\
Datsun_510 & 5079 & 2280 \\
Plym_Horizon & 4482 & 2200 \\
Toyota_Corolla & 3748 & 2200 \\
\hline
\end{tabular}

Every student has got a different schoolwork

\section{Schoolwork}

Student's name: John Goat

Student's ID: 2

\section{Excercise 1}

Calculate the Pearson correlation coefficient between the price and the weight of the following 7 cars:

\begin{tabular}{lrr}
\hline Make & Price & Weight \\
\hline Volvo_260 & 11995 & 3170 \\
VW_Dasher & 7140 & 2160 \\
VW_Scirocco & 6850 & 1990 \\
Olds_Cut_Supr & 5172 & 3310 \\
Plym_Sapporo & 6486 & 2520 \\
Honda_Civic & 4499 & 1760 \\
AMC_Pacer & 4749 & 3350 \\
\hline
\end{tabular}

\section{Schoolwork}

Student's name: Carmen Donkey Student's ID: 3

\section{Excercise 1}

Calculate the Pearson correlation coefficient between the price and the weight of the following 7 cars:

\begin{tabular}{lrr}
\hline Make & Price & Weight \\
\hline Chev_Impala & 5705 & 3690 \\
Volvo_260 & 11995 & 3170 \\
BMW_320i & 9735 & 2650 \\
Plym_Arrow & 4647 & 3260 \\
AMC_Concord & 4099 & 2930 \\
Audi_5000 & 9690 & 2830 \\
Buick_Electra & 7827 & 4080 \\
\hline
\end{tabular}


Step 11: Create a Sar command (in a library) to mark the schoolwork

Plain text file: c:|sar libraries|checkcompute.txt

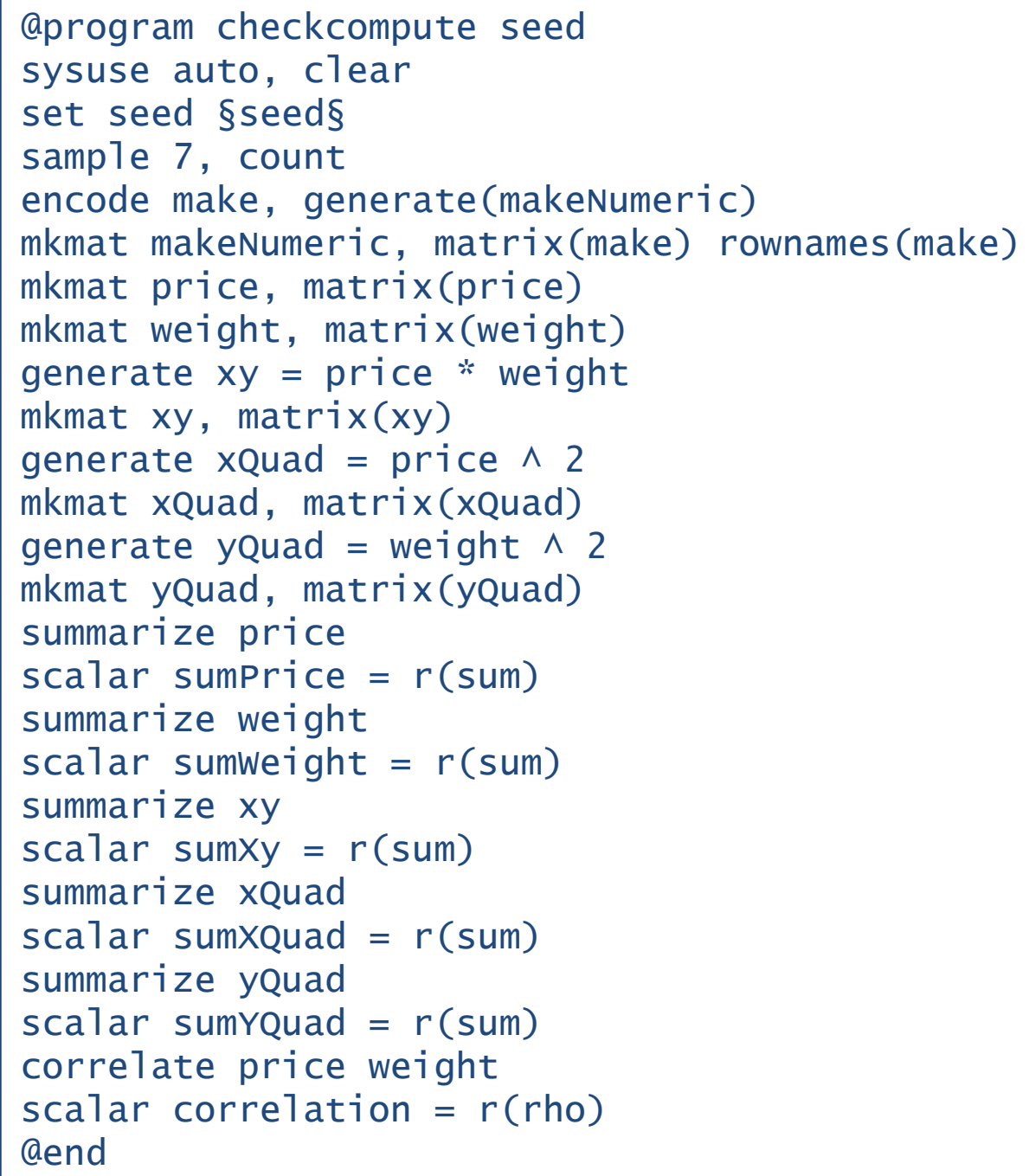




\section{Appendix A - Automatic classwork}

\section{Step 12: Create a checker template which is linked to the students' database}

\section{(Checker)}

Student's name: 《First_Name» «Last_Name»

Student's ID: «D»

\begin{tabular}{|l|r|r|r|r|r|}
\hline Make & $\boldsymbol{x}_{\boldsymbol{i}}$ (Price) & $\boldsymbol{y}_{\boldsymbol{i}}$ (Weight) & $\boldsymbol{x}_{\boldsymbol{i}} \boldsymbol{y}_{\boldsymbol{i}}$ & $\boldsymbol{x}_{\boldsymbol{i}}^{2}$ & $\boldsymbol{y}_{\boldsymbol{i}}^{2}$ \\
\hline Volvo_260 & & & & & \\
\hline VW_Dasher & & & & & \\
\hline VW_Scirocco & & & & & \\
\hline Olds_Cutl_Supr & & & & & \\
\hline Plym_Sapporo & & & & & \\
\hline Honda_Civic & & & & & \\
\hline AMC_Pacer & & & & & \\
\hline Total & & & & & \\
\hline & & & & \\
\hline
\end{tabular}

$$
\begin{aligned}
& r_{x y}=\frac{n \sum x_{i} y_{i}-\sum x_{i} \sum y_{i}}{\sqrt{n \sum x_{i}^{2}-\left(\sum x_{i}\right)^{2}} \sqrt{n \sum y_{i}^{2}-\left(\sum y_{i}\right)^{2}}}= \\
& =\frac{7\left(x_{i}\right)-\left(x_{i}\right)\left(x_{i}\right)}{\sqrt{n\left(x_{i}\right)-\left(x_{i}\right)^{2}} \sqrt{n\left(x_{i}\right)-\left(x_{i}\right)^{2}}}=|x|
\end{aligned}
$$

Comment [sar1]: @loa dlibrary "c: lsar

\begin{tabular}{|c|}
\hline 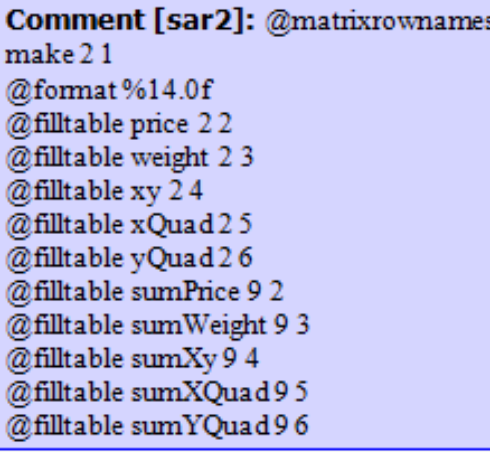 \\
\hline Comment [sar3]: @print sumXy \\
\hline Comment [sar4]: @print sumPrice \\
\hline Comment [sar5]: @print sumWeight \\
\hline Comment [sar6]: @print sumXQuad \\
\hline Comment [sar7]: @print sumPrice \\
\hline Comment [sar8]: @print sumYQuad \\
\hline Comment [sar9]: @print sumWeight \\
\hline $\begin{array}{l}\text { Comment [sar10]: @ format } \% 5.4 \mathrm{f} \\
\text { @print correlation }\end{array}$ \\
\hline
\end{tabular}
librariesicheckcompute.txt"

(a) do checkcompute «ID» 


\section{Appendix A - Automatic classwork}

Step 13: Select the student's ID and launch Sar

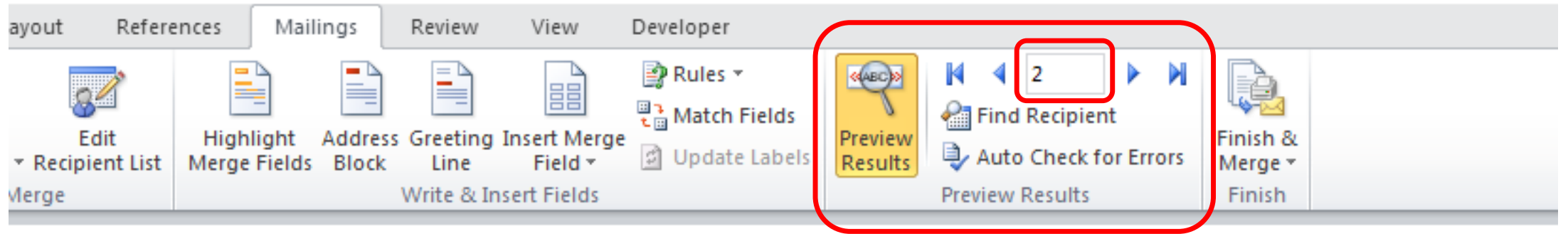

Checker

Student's name: John Goat

Student's ID: 2

\begin{tabular}{lrrrrr}
\hline Make & $\boldsymbol{x}_{\boldsymbol{i}}$ (Price) & $\boldsymbol{y}_{\boldsymbol{i}}$ (Weight) & $\boldsymbol{x}_{\boldsymbol{i}} \boldsymbol{y}_{\boldsymbol{i}}$ & $\boldsymbol{x}_{\boldsymbol{i}}^{\mathbf{2}}$ & $\boldsymbol{y}_{\boldsymbol{i}}$ \\
\hline Volvo_260 & 11995 & 3170 & 38024152 & 143880032 & 10048900 \\
VW_Dasher & 7140 & 2160 & 15422400 & 50979600 & 4665600 \\
VW_Scirocco & 6850 & 1990 & 13631500 & 46922500 & 3960100 \\
Olds_Cutl_Supr & 5172 & 3310 & 17119320 & 26749584 & 10956100 \\
Plym_Sapporo & 6486 & 2520 & 16344720 & 42068196 & 6350400 \\
Honda_Civic & 4499 & 1760 & 7918240 & 20241000 & 3097600 \\
AMC_Pacer & 4749 & 3350 & 15909150 & 22553000 & 11222500 \\
\hline Total & 46891 & 18260 & 124369482 & 353393912 & 50301200 \\
\hline
\end{tabular}

$$
\begin{aligned}
& r_{x y}=\frac{n \sum x_{i} y_{i}-\sum x_{i} \sum y_{i}}{\sqrt{n \sum x_{i}^{2}-\left(\sum x_{i}\right)^{2}} \sqrt{n \sum y_{i}^{2}-\left(\sum y_{i}\right)^{2}}}= \\
& =\frac{7(124369482)-(46891)(18260)}{\sqrt{n(353393912)-(46891)^{2}} \sqrt{n(50301200)-(18260)^{2}}}=0.2003
\end{aligned}
$$


Conclusions about automatic schoolwork:

- You can discourage students from copying during classwork

- By using "mailings» functions of Word, you can send by email to your students:

- automatic homework

- automatic solutions with calculations and formulas

- You can manage exercises in the classroom encouraging students to work on their own 


\section{The problem}

If the number of observations on which an estimate is based falls below a minimum criterion, a warning should accompany the estimate

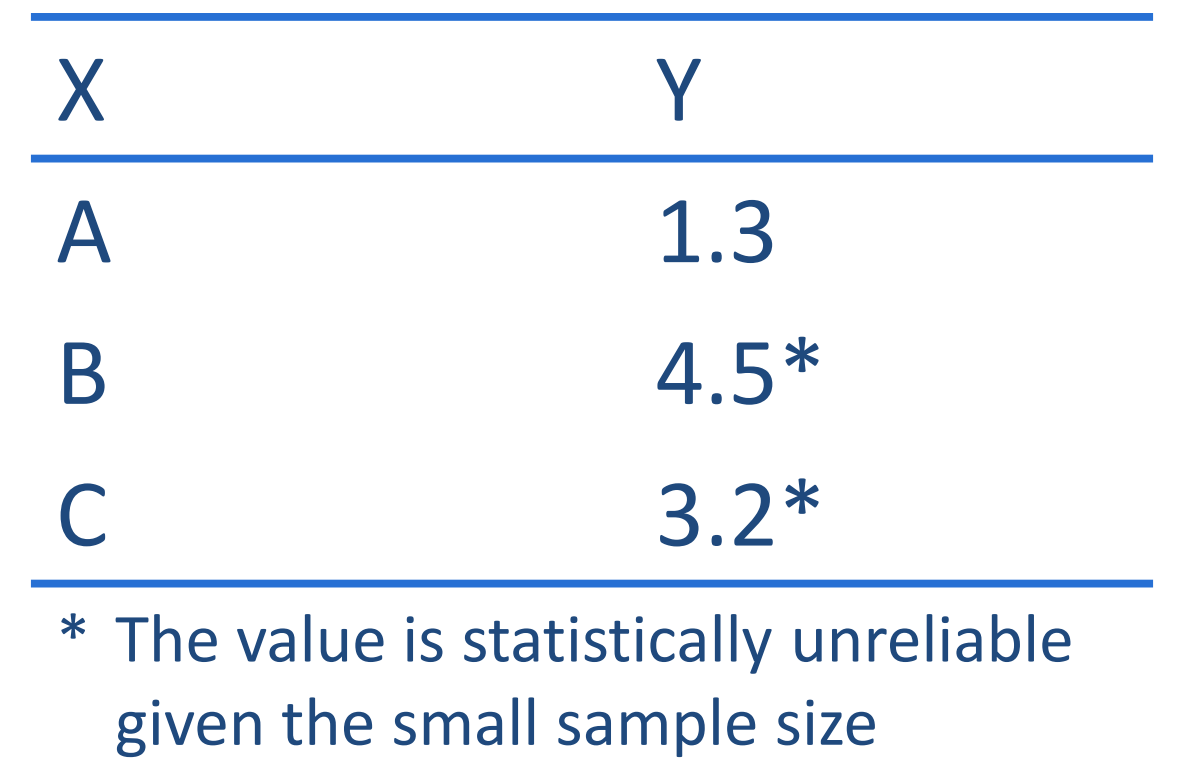

\section{The solution}

An automatic document created with Sar where an asterisk is added to the reported estimates which are based on a low number of observations 


\section{Example:}

A report with mean wage by industry from the nlsw88.dta dataset. We want to highlight estimates wich are based on less then $\mathbf{3 0}$ observations
- sysuse n1sw88

(NLSW, 1988 extract)

- mean wage, over(industry)

(output suppressed)

- $\operatorname{matrix} \mathrm{N}=\mathrm{e}\left(\mathrm{N}_{\mathrm{N}}\right)$ ‘

- matrix list $\mathrm{N}$

This matrix contains the number of observations used in estimating mean wages by industry $N[12,1]$

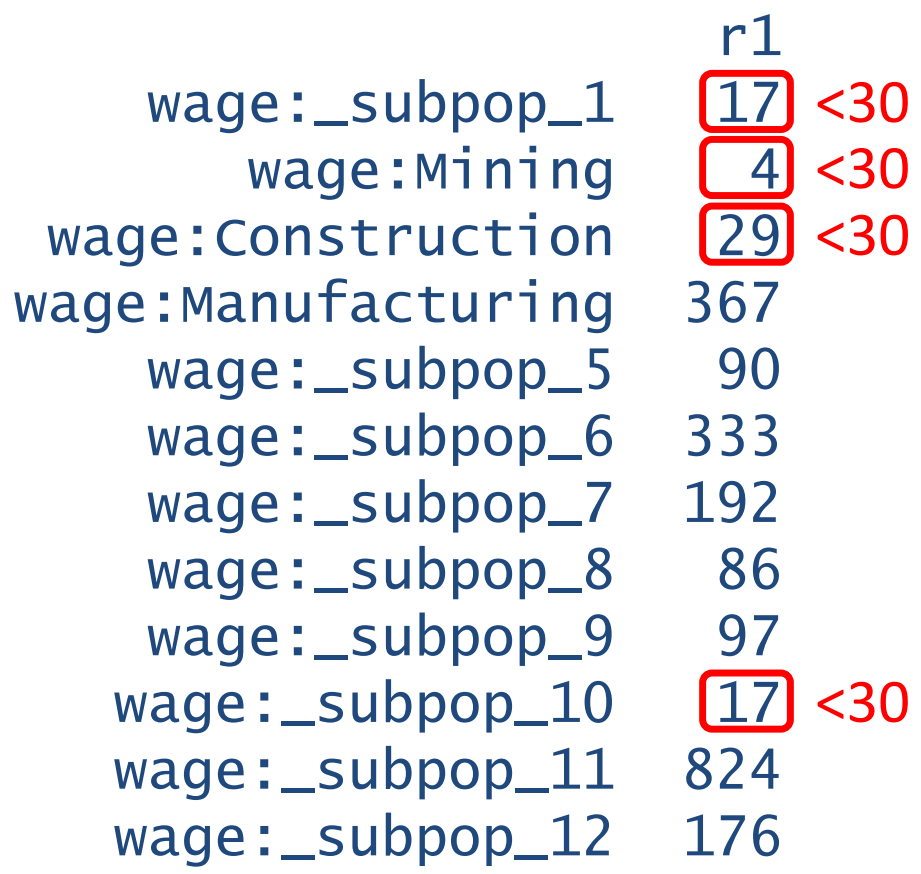


Step 1: create the gen 1ownumbermatrix program and put it in a valid ADO path (See the next slide to know how the gen lownumbermatrix program works)

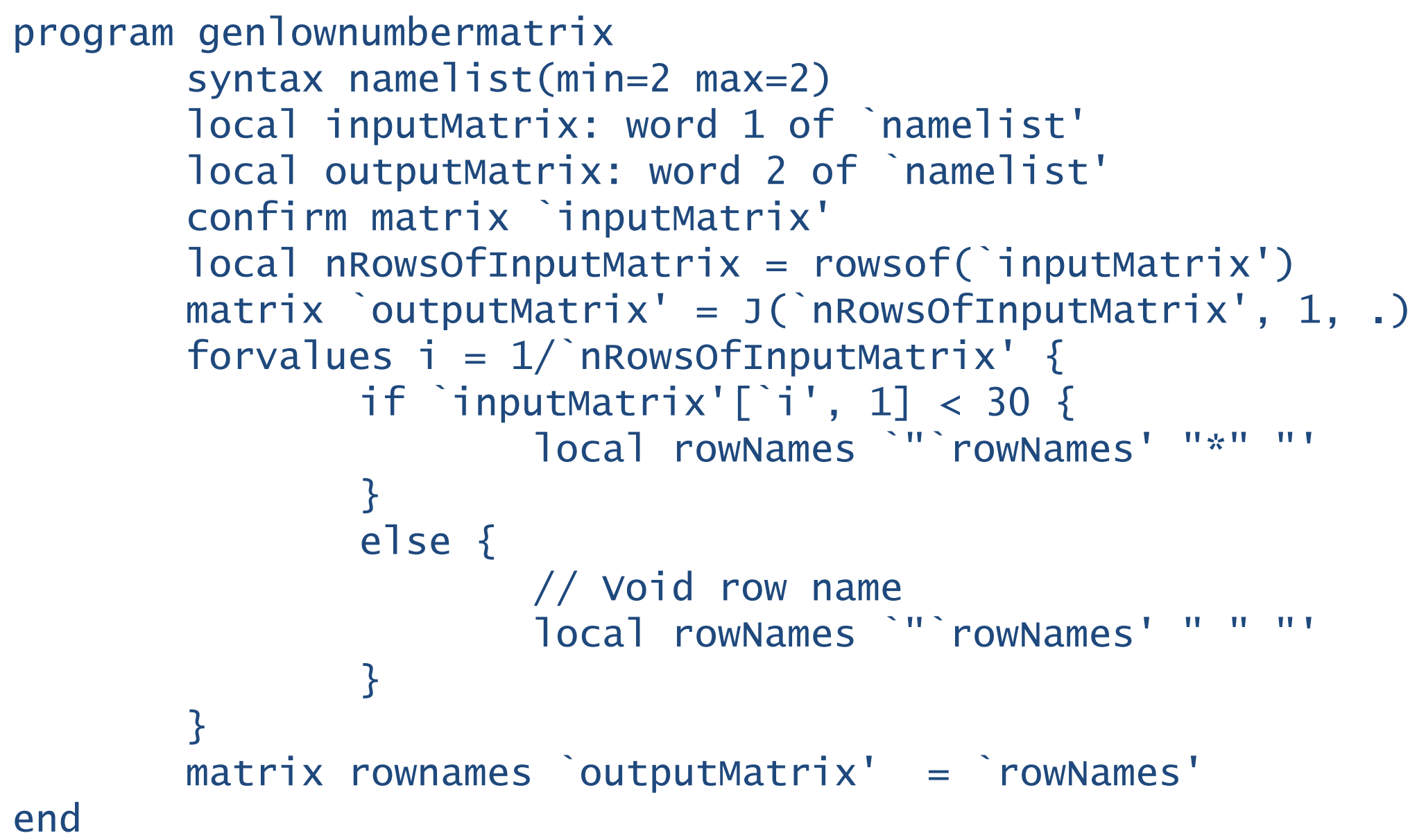


How the gen lownumbermatrix works

\author{
Syntax \\ genlownumbermatrix inputMatrix outputMatrix
}

\title{
Description
}

The gen lownumbermatrix program creates an output matrix in which the matrix row names are asterisks if the corresponding row value in the input matrix is less than 30

\section{Example}

- $\operatorname{matrix} \mathrm{N}=(12 \backslash 32 \backslash 8)$

- genlownumbermatrix $\mathrm{N}$ lown

- matrix 1ist lown

lown $[3,1]$

c1

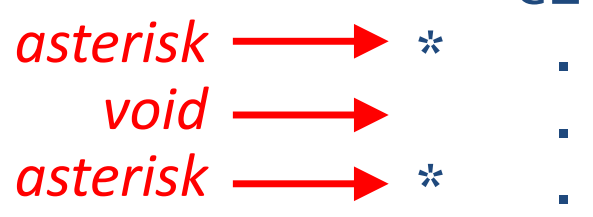


Step 2: create a Sar automatic report which calls the gen 1 ownumbermatrix program

Mean wage by industry

\begin{tabular}{l|r}
\hline Industry & Wage \\
\hline $\mathrm{Ag} /$ Forestry/Fisheries & $5.6^{*}$ \\
\hline Mining & $15.3^{*}$ \\
\hline Construction & $7.6^{*}$ \\
\hline Manufacturing & 7.5 \\
\hline Transport/Comm/Utility & 11.4 \\
\hline Wholesale/Retail Trade & 6.1 \\
\hline Finance/Ins/Real Estate & 9.8 \\
\hline Business/Repair Svc & 7.5 \\
\hline Personal Services & 4.4 \\
\hline Entertainment/Rec Svc & $6.7^{*}$ \\
\hline Professional Services & 7.9 \\
\hline Public Administration & 9.1 \\
\hline
\end{tabular}

(*) number of observation is less than 30
Comment [sar1]: sysuse nlsw8 8 mean wage, over(industry)

matrix wage $=\mathrm{e}(\mathrm{b})^{\prime}$

matrix $\mathrm{N}=\mathrm{e}(\mathrm{N})^{\prime}$

genlownumbermatrix $\mathrm{N}$ low $\mathrm{N}$
Comment [sar2]: @format \%3.1f @ filltable wage 22 @matrixrownames lowN 23 
Conclusions about highlighting subgroups with low number of observations:

- Warning about statistics which are based on a small sample is a good practice

- Sar can be used to automate numbers and text as well (asterisks for example)

- You can improve the gen 1ownumbermatrix program by:

- adding an argument which represents the threshold (a fixed threshold of 30 was used in the example)

- adding an argument which represents an alternative symbol to asterisk 
Sar notifies you when an error occurred

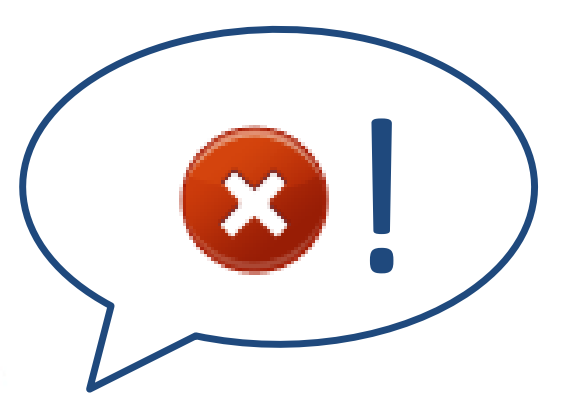

\section{Sar}




\section{[Mybeautiful-reporț}

\section{Comment [sar1]: sysuse auto}

In this report I comment some.

summary statistics from the

auto.dta dataset. 9

The mean price is $(X)$. I

Comment [sar2]: summarize price

@format $\% 5.1 \mathrm{f}$

$@ \operatorname{printtt} r($ mean $)$

Stata automatic report

Error in comment 2, paragraph 3.

Stata command error.

Warning: elaboration was stopped where error occurred.
The command in the third paragraph of comment 2 is wrong («@printtt» was typed instead of «@print») 
Sar halts the execution of the commands where the error occurres: you can open the Stata window to debug your Sar session

\footnotetext{
- count
}

0

* Sar internally uses the previous command "count" to synchronize with stata.

* Don't worry about it.

sysuse auto

(1978 Automobile Data)

summarize price

\begin{tabular}{|c|c|c|c|c|c|}
\hline Variable | & Obs & Mean & Std. Dev. & Min & $\operatorname{Max}$ \\
\hline price & 74 & 6165.257 & 2949.496 & 3291 & 15906 \\
\hline
\end{tabular}

confirm numeric format $\% 5.1 f$

aprinttt $r($ mean)

unrecognized command: @ invalid command name 
Tip: use @viewlog and @interact to debug

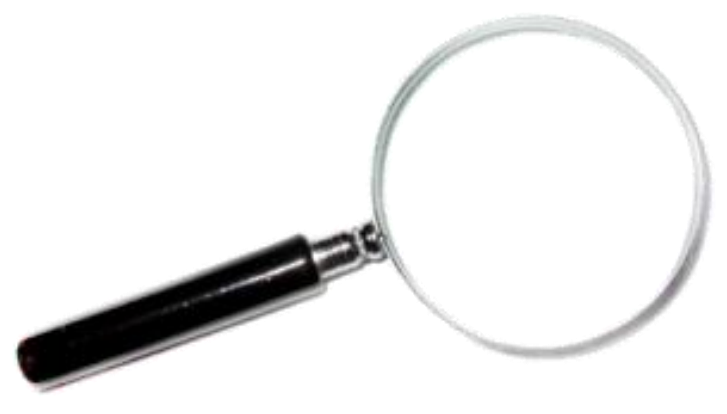

- Qviewlog

When used (it does not matter in which Sar comment) it leaves the Stata window open after Sar is executed, so you can see the log of your session

- Qinteract

It halts Sar execution and makes Stata at your disposal 


\section{Appendix D - Syntax and description of the Sar commands}

\section{abeginstring}

\section{Syntax}

abeginstring \#string\#

\section{Description}

The abeginstring command sets the string of characters you want to put before the numerical outputs of the Qfil1table command.

The string must be specified between two sharps (\#).

See also the @endstring command.

\section{@cleartable}

\section{Syntax}

acleartable

\section{Description}

The @cleartable command clears the table associated with the comment where the command is written. It can only be used within Word comments associated with a single table.

The command has no arguments.

\section{@do}

\section{Syntax}

Qdo Sarprogram

\section{Description}

The @do command executes a program previously loaded by the @1oadlibrary command or defined in a Word comment through the @program/@end paradigm.

The SarProgram argument specifies the program which has to be executed. 


\section{Appendix D - Syntax and description of the Sar commands}

\section{Qendstring}

\section{Syntax}

@endstring \#string\#

\section{Description}

The Qendstring command sets the string of characters you want to place after the numerical outputs of the afi 11 table command.

The string must be specified between two sharps (\#).

See also the @beginstring command syntax and description.

\section{Qfi11table}

\section{Syntax}

@fil1table stataData startingRow startingco7 [rowstep co7step]

\section{Description}

The afi11table command inserts values from matrices in a table, Stata results, scalars and macros given by the StataData argument in a Word table. It can be used only in Word comments associated with a single table.

StataData is the data retrieved from the Stata environment used by the command to fill the table. It can be a matrix, a Stata result, a scalar or a macro.

startingRow and startingCol indicate, respectively, the row and the column of the table cell from which StataData begins to be printed. They have to be nonzero integers. If these values are negative, -1 means last row/column, -2 means second-last row/second-last column and so on.

rowStep and colStep indicate, respectively, how many rows (columns) have to be skipped, between a row (column) and the next row (column), filling the table. When rowStep/colStep equals 0 , no blank row/column is left between printed rows/columns. When rowStep/colstep equals 1, a blank row/column is left between printed rows/columns. Generally, if rowStep/colStep equals $n$, then $n$ blank rows/columns are left between printed rows/columns. These arguments are optional and they have to be non-negative integers. 


\section{Appendix D - Syntax and description of the Sar commands}

\section{Qformat}

\section{Syntax}

aformat \%fmt

\section{Description}

The @format command sets the numerical format of the output obtained by @print and @ fi 11 table commands. The set numerical format is preserved for the following @print and @fi11table commands.

The \%fmt argument has to be a numerical format written using the same rules used in the Stata format command (See he1p format in Stata).

\section{Qinteract}

\section{Syntax}

ainteract

\section{Description}

The @interact command haltes the execution of Sar to make Stata at your disposal. So you can use Stata, interact with it and create data objects (like scalars or matrices) that will be available in the Sar environment after your Stata session has been closed. Remember to not manually close the Stata window: this will cause the crash of Sar. You have to return to Word, where you will find a dialog window with a button to close Stata. The command has no arguments.

\section{@1oad1ibrary}

\section{Syntax}

@1oad1ibrary "pathofTheLibraryfi 7e"

\section{Description}

The @1oad1 ibrary command loads programs defined in a Sar library file.

The path of the Sar library file has to be specified in the pathOfTheLibraryFile argument. 


\section{Appendix D - Syntax and description of the Sar commands}

\section{@matrixcolnames and @matrixrownames}

\section{Syntax}

@matrixcolnames StataMatrix stratingRow startingco7 [co7step]

amatrixrownames StataMatrix startingRow startingCo7 [rowstep]

\section{Description}

The @matrixcolnames and @matrixrownames commands fill a Word table with, respectively, row-names and column-names of a Stata matrix. They can be used only in Word comments associated with a single table.

StataData is the matrix retrieved from the Stata environment whose matrix row-names are printed by @matrixrownames and whose matrix column-names are printed by @matrixcolnames. This argument has to be a matrix.

startingRow and startingCol indicate, respectively, the row and the column of the table cell from which the rownames/column-names of StataMatrix begin to be printed. They have to be nonzero integers. If these values are negative, -1 will indicate the last row/column, -2 will indicate the second-last row/second-last column and so on. colstep is an optional argument for @matrixcolnames. It indicates the column step according to the table is filled. The default value is 0 . It has to be a non-negative integer.

rowstep is an optional argument of @matrixrownames. It indicate the row step according to the table is filled. The default value is 0 . It has to be a non-negative integer.

\section{aprint}

\section{Syntax}

aprint Statava7ue

\section{Description}

The aprint command, launched from a Word comment associated with a portion of text (a temporary text placeholder in the Sar jargon), replaces its placeholder with the value of a Stata result, a scalar or a macro retrieved from the Stata environment. The aprint command can not be used in a Word comment associated with a table. The StataValue argument must be a Stata result, a scalar or a macro. 


\section{Appendix D - Syntax and description of the Sar commands}

\section{@program/@end paradigm}

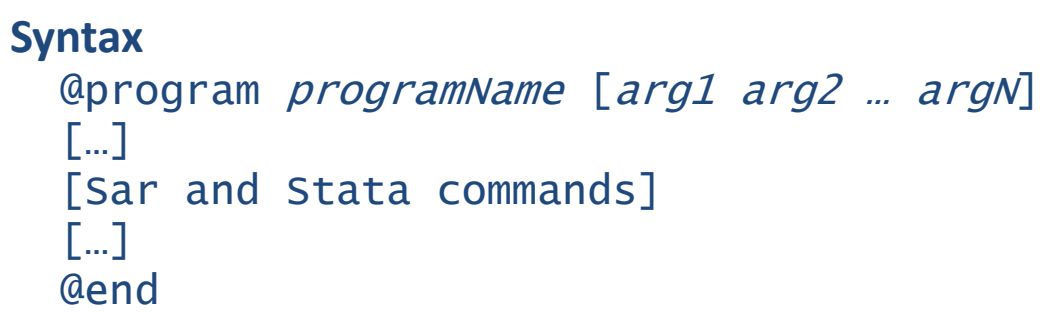

\section{Description}

The @program/@end paradigm is used to define a Sar program. This paradigm can be used in a Word comment or in a Sar library. Sar programs are, roughly speaking, a list of Sar and Stata commands. This list of commands is defined between the aprogram and the @do commands. After the commands are loaded in the Sar environment, they can be executed through the ado command.

The programName argument is used to set the name of the program.

The optional arguments $\arg 1, \arg 2, \ldots, \arg N$ specify the arguments of the program defined by the @program/@end paradigm. When you want to use the values passed as arguments in your program, you have to use the $\S a r g 1 \S$, $\S a r g 2 \S, \ldots, \S a r g N \S$ callbacks inside your program code: before executing the program Sar replaces every callback with the corresponding values of arguments.

The @end command closes a program definition. It has no arguments.

The following commands can not be used in a Sar program: @do, @loadlibrary, @interact and the @program/@end paradigm. 


\section{Appendix D - Syntax and description of the Sar commands}

\section{Qresetstring}

\section{Syntax}

aresetstring

\section{Description}

The aresetstring command sets to an empty string the string of characters which is putted before and after the numerical outputs of the aprint and afil1table commands: when the aresetstring command is used no characters are added before or after the numerical output. It's equivalent to the couple of command abeginstring \#\# and @endstring \#\#.

The command has no arguments.

See also @beginstring and @endstring syntax and description.

\section{Qviewlog}

\section{Syntax}

Qviewlog

\section{Description}

The @viewlog command asks Sar to leave the Stata window open after the Sar macro was executed. This can be useful to look at the log created by Stata computations.When @viewlog is used, in whatever word comment, a dialog window is opened after the execution of the Sar macro, allowing you to close the Stata Window and terminate the Sar macro.

The command has no arguments. 


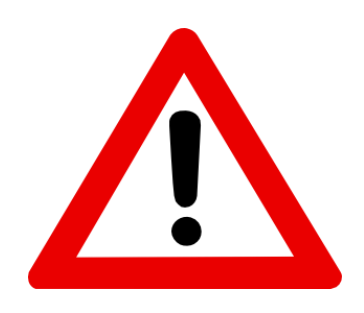

The user is the only responsible for the accuracy of the statistical analysis and for possible damages caused by Sar. It's strongly recommended to save the Word document before you launch Sar. 